\title{
Supporting Information for: Open for bismuth: main group metal-to-ligand charge transfer
}

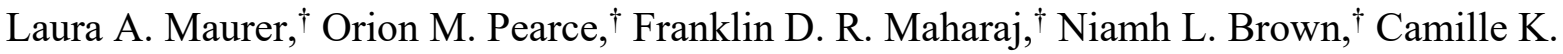
Amador, ${ }^{\dagger}$ Niels H. Damrauer, ${ }^{\dagger},+$ and Michael P. Marshak ${ }^{*, \dagger}, \dagger$

†Department of Chemistry, University of Colorado Boulder, Boulder, Colorado 80309, United States

Renewable and Sustainable Energy Institute, Boulder, Colorado 80309, United States

michael.marshak@colorado.edu

Department of Chemistry, University of Colorado Boulder, Boulder Colorado 80309, United States

\section{Contents}

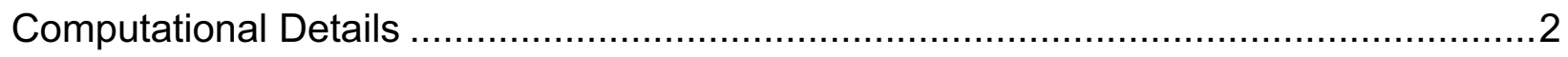

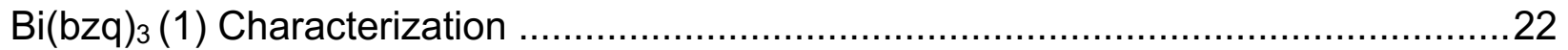

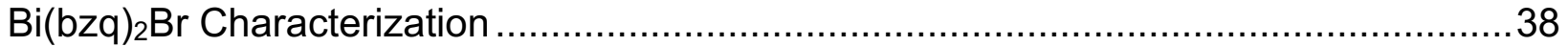

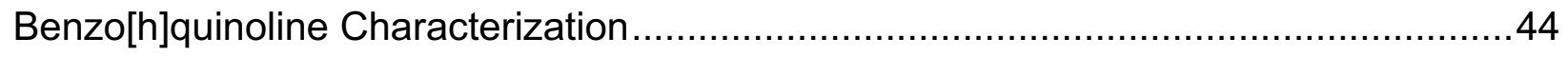

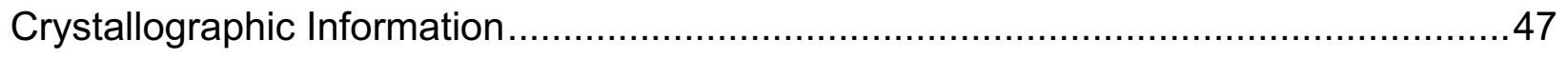

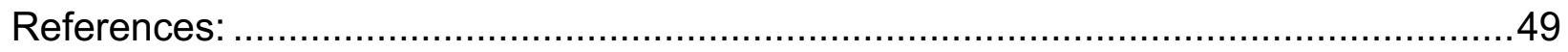




\section{Computational Details}

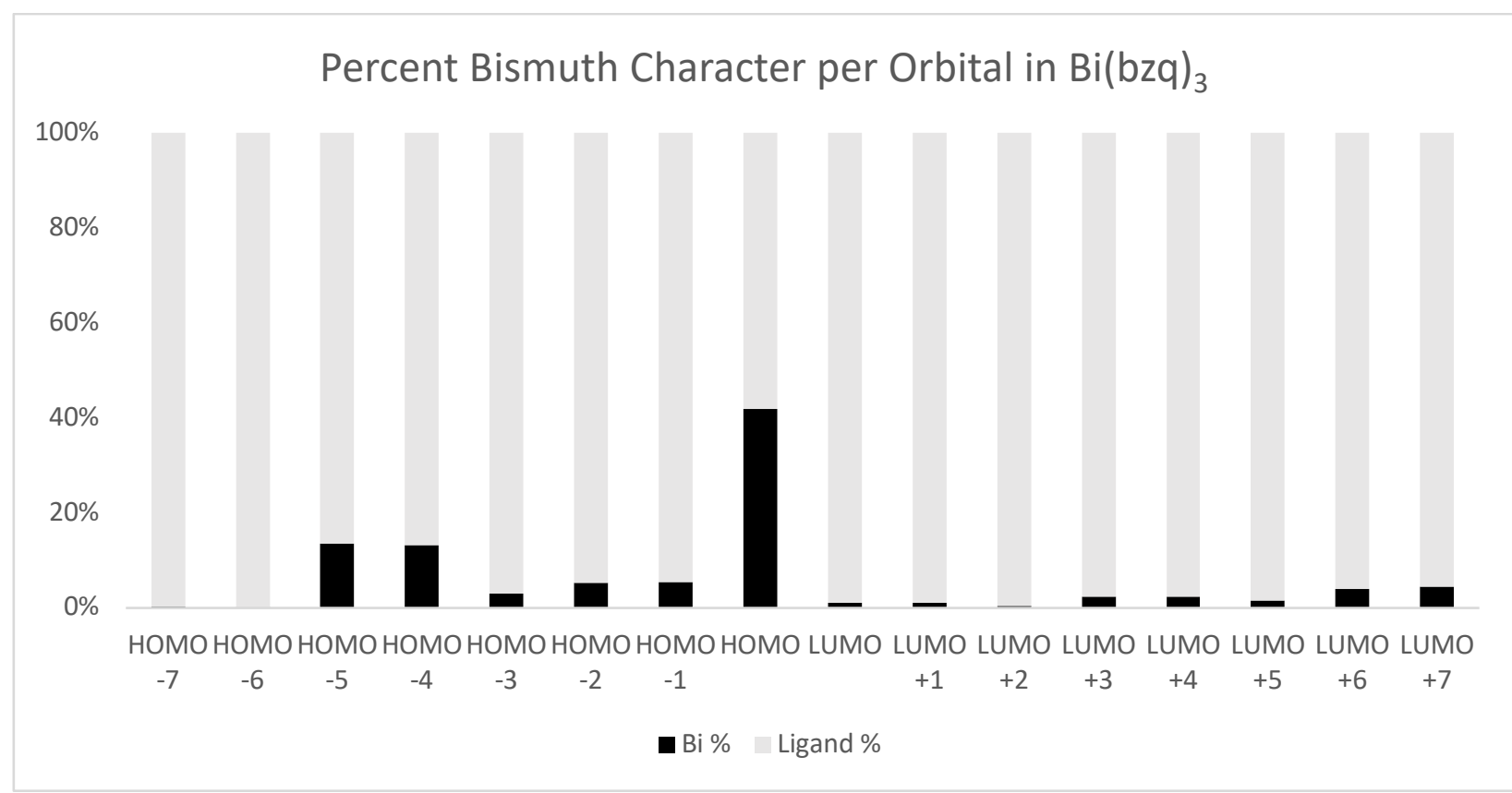

Figure S1. Theoretical bismuth percentage in calculated orbitals

Electron percentage of the compound in various orbitals. The large shift in bismuth character between the LUMO and HOMO suggests an MLCT. 


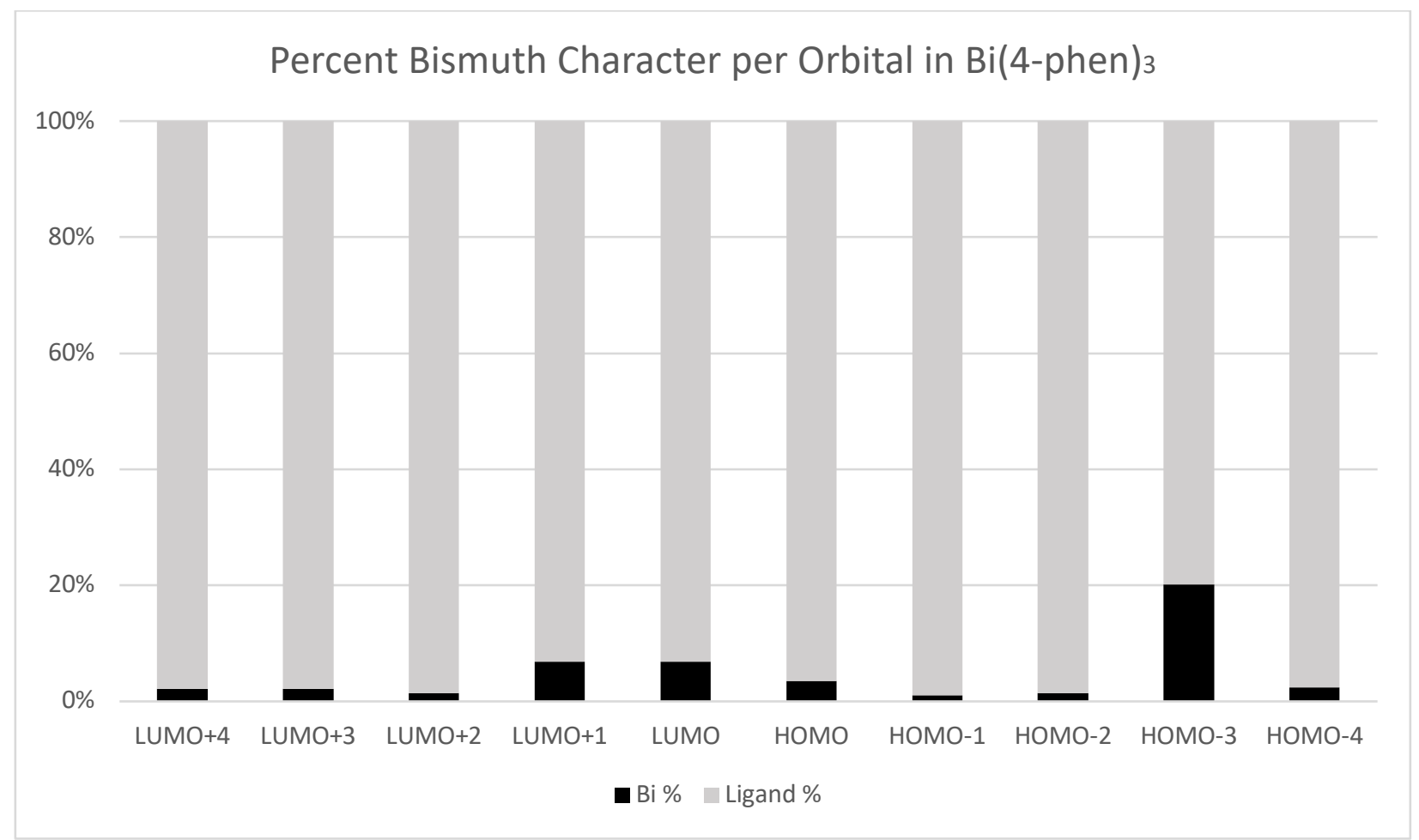

Figure S2. Theoretical bismuth percentage in calculated orbitals of Bi(4-phen $)_{3}$ Electron percentage of the compound in various orbitals. The large shift in bismuth character between the LUMO and HOMO suggests an MLCT. 
Table S1. Calculated orbital energies of $\mathrm{Bi}(\mathrm{bzq})_{3}(\mathbf{1})$ around the HOMO and LUMOSinglet Multiplicity

\begin{tabular}{|c|c|c|c|}
\hline Energy (eV) & $\begin{array}{c}\text { Electron } \\
\text { Occupation }\end{array}$ & Bi \% & $\begin{array}{c}\text { Ligand } \\
\%\end{array}$ \\
\hline-0.2868 & 0 & $5.6 \%$ & $94.4 \%$ \\
\hline-0.363 & 0 & $6.6 \%$ & $93.4 \%$ \\
\hline-0.9881 & 0 & $4.2 \%$ & $95.8 \%$ \\
-0.9897 & 0 & $4.4 \%$ & $95.6 \%$ \\
\hline-1.0658 & 0 & $4.0 \%$ & $96.0 \%$ \\
\hline-1.8618 & 0 & $1.6 \%$ & $98.4 \%$ \\
\hline-1.9555 & 0 & $2.4 \%$ & $97.6 \%$ \\
-1.9575 & 0 & $2.4 \%$ & $97.6 \%$ \\
\hline-2.2559 & 0 & $0.4 \%$ & $99.6 \%$ \\
\hline-2.2980 & 0 & $1.0 \%$ & $99.0 \%$ \\
\hline-2.2996 & 0 & $1.0 \%$ & $99.0 \%$ \\
\hline-4.7439 & 2 & $41.9 \%$ & $58.1 \%$ \\
\hline-5.2703 & 2 & $5.5 \%$ & $94.5 \%$ \\
-5.2735 & 2 & $5.3 \%$ & $94.7 \%$ \\
\hline-5.3586 & 2 & $3.0 \%$ & $97.0 \%$ \\
\hline-5.4164 & 2 & $13.2 \%$ & $86.8 \%$ \\
\hline-5.4231 & 2 & $13.6 \%$ & $86.4 \%$ \\
\hline-5.7702 & 2 & $0.1 \%$ & $99.9 \%$ \\
\hline-5.7712 & 2 & $0.2 \%$ & $99.8 \%$ \\
\hline-5.7853 & 2 & $0.3 \%$ & $99.7 \%$ \\
\hline-6.2631 & 2 & $17.7 \%$ & $82.3 \%$ \\
\hline & & & \\
\hline
\end{tabular}

Orbital energies within $k_{b} T$ at room temperature $(0.025 \mathrm{eV})$ are grouped together to indicate degeneracy. The percent bismuth and ligand character are given for each orbital. 
Table S2. Selected Spin-Allowed Transitions (Singlet Ground to Singlet Excited) from TD-DFT of Bi(bzq) $)_{3}(1)$

\begin{tabular}{|c|c|c|c|c|c|}
\hline State & $\begin{array}{l}\text { Energy } \\
\text { / eV }\end{array}$ & $\lambda / \mathrm{nm}$ & $\begin{array}{l}\text { Oscillator } \\
\text { Strength }\end{array}$ & & Assignment \\
\hline 1 & 2.474 & 501.1 & 0.011 & HOMO $\rightarrow$ LUMO & MLCT \\
\hline 2 & 2.476 & 501.8 & 0.011 & HOMO $\rightarrow$ LUMO+1 & MLCT \\
\hline 3 & 2.513 & 493.4 & 0.002 & HOMO $\rightarrow$ LUMO+2 & MLCT \\
\hline 4 & 2.817 & 440.1 & 0.017 & HOMO $\rightarrow$ LUMO+3 & MLCT \\
\hline 5 & 2.819 & 439.8 & 0.017 & $\mathrm{HOMO} \rightarrow \mathrm{LUMO}+4$ & MLCT \\
\hline 6 & 2.912 & 425.7 & 0.000 & HOMO $\rightarrow$ LUMO+5 & MLCT \\
\hline 7 & 2.977 & 416.5 & 0.000 & $\begin{array}{c}\text { HOMO-1 } \rightarrow \text { LUMO } \\
\text { HOMO- } 2 \rightarrow \text { LUMO+2 }\end{array}$ & pi-pi ${ }^{*}$ \\
\hline 8 & 2.995 & 414.0 & 0.001 & $\begin{array}{c}\text { HOMO-2 } \rightarrow \text { LUMO } \\
\text { HOMO- } 1 \rightarrow \text { LUMO+2 } \\
\text { HOMO- } 2 \rightarrow \text { LUMO+2 }\end{array}$ & pi-pi* \\
\hline 9 & 2.996 & 413.8 & 0.002 & $\begin{array}{c}\text { HOMO-2 } \rightarrow \text { LUMO+2 } \\
\text { HOMO- } 2 \rightarrow \text { LUMO+2 } \\
\text { HOMO- } 1 \rightarrow \text { LUMO }\end{array}$ & pi-pi* \\
\hline 10 & 3.048 & 406.8 & 0.001 & $\begin{array}{c}\text { HOMO-3 } \rightarrow \text { LUMO } \\
\text { HOMO-3 } \rightarrow \text { LUMO+1 } \\
\text { HOMO- } 1 \rightarrow \text { LUMO+2 }\end{array}$ & pi-pi* \\
\hline 11 & 3.049 & 406.7 & 0.001 & $\begin{array}{c}\text { HOMO-3 } \rightarrow \text { LUMO } \\
\text { HOMO-3 } \rightarrow \text { LUMO+1 } \\
\text { HOMO- } 2 \rightarrow \text { LUMO+2 }\end{array}$ & pi-pi ${ }^{*}$ \\
\hline 12 & 3.054 & 405.9 & 0.002 & $\begin{array}{c}\text { HOMO-3 } \rightarrow \text { LUMO+2 } \\
\text { HOMO- } 2 \rightarrow \text { LUMO } \\
\text { HOMO- } 1 \rightarrow \text { LUMO+1 }\end{array}$ & pi-pi* \\
\hline 13 & 3.128 & 396.4 & 0.000 & $\begin{array}{c}\text { HOMO-4 } \rightarrow \text { LUMO } \\
\text { HOMO-4 } \rightarrow \text { LUMO+1 }\end{array}$ & sigma(b)-pi* \\
\hline 14 & 3.134 & 395.6 & 0.001 & $\begin{array}{l}\text { HOMO-5 } \rightarrow \text { LUMO+1 } \\
\text { HOMO- } 4 \rightarrow \text { LUMO } \\
\text { HOMO } 4 \rightarrow \text { LUMO }+1\end{array}$ & sigma(b)-pi* \\
\hline 15 & 3.135 & 395.5 & 0.001 & $\begin{array}{c}\text { HOMO-5 } \rightarrow \text { LUMO } \\
\text { HOMO-5 } \rightarrow \text { LUMO+1 } \\
\text { HOMO-4 } \rightarrow \text { LUMO+2 }\end{array}$ & sigma(b)-pi* \\
\hline 16 & 3.157 & 392.7 & 0.001 & $\begin{array}{c}\text { HOMO-5 } \rightarrow \text { LUMO } \\
\text { HOMO- } 5 \rightarrow \text { LUMO+1 } \\
\text { HOMO- } 4 \rightarrow \text { LUMO } \\
\text { HOMO- } 4 \rightarrow \text { LUMO+1 }\end{array}$ & sigma(b)-pi ${ }^{*}$ \\
\hline 17 & 3.180 & 389.8 & 0.008 & $\begin{array}{l}\text { HOMO-5 } \rightarrow \text { LUMO+1 } \\
\text { HOMO- } 4 \rightarrow \text { LUMO+2 }\end{array}$ & sigma(b)-pi* \\
\hline 18 & 3.186 & 389.2 & 0.009 & HOMO-5 $\rightarrow$ LUMO+2 & sigma(b)-pi* \\
\hline 19 & 3.314 & 374.2 & 0.003 & $\begin{array}{l}\text { HOMO-2 } \rightarrow \text { LUMO+4 } \\
\text { HOMO- } 1 \rightarrow \text { LUMO+3 }\end{array}$ & pi-pi ${ }^{*}$ \\
\hline 20 & 3.337 & 371.6 & 0.018 & $\begin{array}{l}\text { HOMO- } 2 \rightarrow \text { LUMO }+3 \\
\text { HOMO } 1 \rightarrow \text { LUMO }+4\end{array}$ & pi-pi* \\
\hline
\end{tabular}


$\underline{\text { Table S3. Orbital Surfaces of Bi(bzq })_{3}(1) \text { and descriptions }}$

\begin{tabular}{|l|l|}
\hline & Description \\
\hline & Orbital 186, LUMO+5, bzq-T* \\
\hline
\end{tabular}




Orbital 183, LUMO+2, bzq-T*




Orbital 179, HOMO-1, bzq




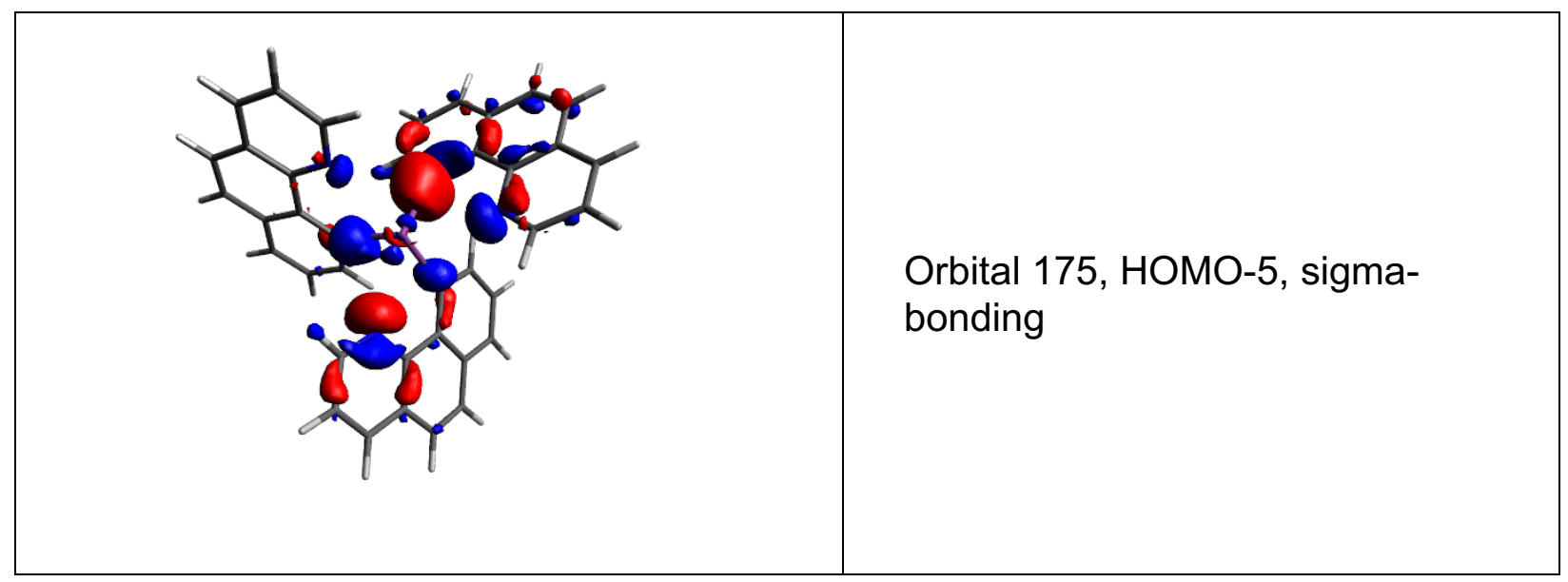


Table S4. Singlet (spin-allowed) transitions Bi(bzq) 3 $_{3}(\mathbf{1})$ from TD-DFT

TD-DFT/TDA EXCITED STATES (SINGLETS)

the weight of the individual excitations are printed if larger than 0.01

STATE 1: $\mathrm{E}=0.090922 \mathrm{au} \quad 2.474 \mathrm{eV} \quad 19955.0 \mathrm{~cm}^{* *}-1$

$180 \mathrm{a}->181 \mathrm{a}: 0.984352(\mathrm{c}=-0.99214503)$

STATE 2: $\mathrm{E}=0.090975 \mathrm{au} \quad 2.476 \mathrm{eV} \quad 19966.7 \mathrm{~cm}^{* *}-1$ $180 a->182 a$ : $0.984343(c=-0.99214063)$

STATE 3: $E=0.092351 \mathrm{au} \quad 2.513 \mathrm{eV} \quad 20268.6 \mathrm{~cm}^{* *}-1$ $180 \mathrm{a}->183 a$ : $0.981560(\mathrm{c}=-0.99073687)$

STATE 4: $\mathrm{E}=0.103531 \mathrm{au} \quad 2.817 \mathrm{eV} \quad 22722.4 \mathrm{~cm}^{\star *}-1$ $180 \mathrm{a}->184 \mathrm{a}: 0.962503(\mathrm{c}=0.98107216)$ $180 \mathrm{a}->185 \mathrm{a}: 0.023753(\mathrm{c}=-0.15412097)$

STATE 5: $\mathrm{E}=0.103611 \mathrm{au} \quad 2.819 \mathrm{eV} \quad 22739.9 \mathrm{~cm}^{* *}-1$ $180 \mathrm{a}->184 \mathrm{a}: 0.023729$ (c= 0.15404095$)$ $180 \mathrm{a}->185 \mathrm{a}: 0.962464(\mathrm{c}=0.98105272)$

STATE 6: $\mathrm{E}=0.107029 \mathrm{au} \quad 2.912 \mathrm{eV} \quad 23490.1 \mathrm{~cm}^{\star *}-1$ $180 \mathrm{a}->186 \mathrm{a}: 0.982262(\mathrm{c}=-0.99109137)$

STATE 7: $\mathrm{E}=0.109401 \mathrm{au} \quad 2.977 \mathrm{eV} \quad 24010.7 \mathrm{~cm}^{* *}-1$ $178 \mathrm{a}->181 \mathrm{a}: 0.048389(\mathrm{c}=0.21997552)$ $178 \mathrm{a}->182 \mathrm{a}: 0.363909(\mathrm{c}=-0.60324831)$ $179 \mathrm{a}->181 \mathrm{a}: \quad 0.534374(\mathrm{c}=0.73100913)$ $179 \mathrm{a}->182 \mathrm{a}: \quad 0.049361(\mathrm{c}=0.22217425)$

STATE 8: $\mathrm{E}=0.110052 \mathrm{au} \quad 2.995 \mathrm{eV} \quad 24153.5 \mathrm{~cm}^{* *}-1$ $177 \mathrm{a}->181 \mathrm{a}: 0.010474(\mathrm{c}=-0.10234177)$ $177 \mathrm{a}->182 \mathrm{a}: \quad 0.021122(\mathrm{c}=0.14533256)$ $178 \mathrm{a}->181 \mathrm{a}: \quad 0.332323(\mathrm{c}=0.57647461)$ $179 \mathrm{a}->182 \mathrm{a}: 0.354097(\mathrm{c}=-0.59506058)$ $179 a->183 a ： 0.253091(c=0.50308198)$

STATE 9: $E=0.110106 \mathrm{au} \quad 2.996 \mathrm{eV} \quad 24165.5 \mathrm{~cm}^{* *}-1$ $177 a->181 a: 0.025594(c=-0.15998076)$ $177 \mathrm{a}->182 \mathrm{a}: 0.010173(\mathrm{c}=-0.10085968)$ $178 \mathrm{a} \rightarrow \mathrm{181a}: \quad 0.013254(\mathrm{c}=0.11512413)$ $178 \mathrm{a}->182 \mathrm{a}: \quad 0.420901(\mathrm{c}=-0.64876872)$ $178 \mathrm{a}->183 \mathrm{a}: 0.245660(\mathrm{c}=-0.49564128)$ $179 a->181 a: 0.263958(c=-0.51376857)$ $179 a->183 a ： 0.010998(c=-0.10487005)$

STATE 10: $\mathrm{E}=0.111999 \mathrm{au} \quad 3.048 \mathrm{eV} \quad 24581.0 \mathrm{~cm}^{* *}-1$ $177 \mathrm{a}->181 \mathrm{a}: 0.267246(\mathrm{c}=0.51695870)$ $177 \mathrm{a}->182 \mathrm{a}: \quad 0.234208(\mathrm{c}=-0.48395062)$ $178 \mathrm{a}->181 \mathrm{a}: \quad 0.035659(\mathrm{c}=-0.18883580)$ $178 \mathrm{a}->183 \mathrm{a}: \quad 0.084472(\mathrm{c}=-0.29064046)$ $179 \mathrm{a}->182 \mathrm{a}: \quad 0.022459(\mathrm{c}=0.14986442)$ $179 \mathrm{a}->183 \mathrm{a}: \quad 0.338510(\mathrm{c}=0.58181597)$ 


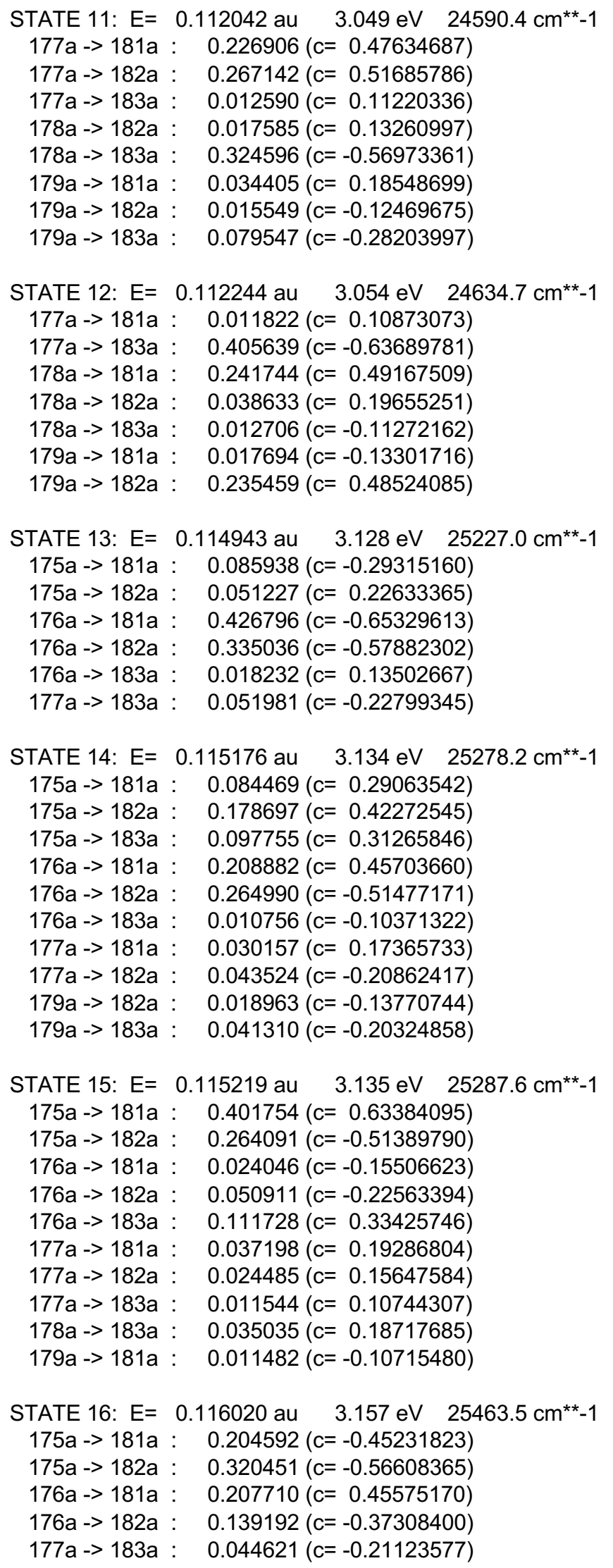




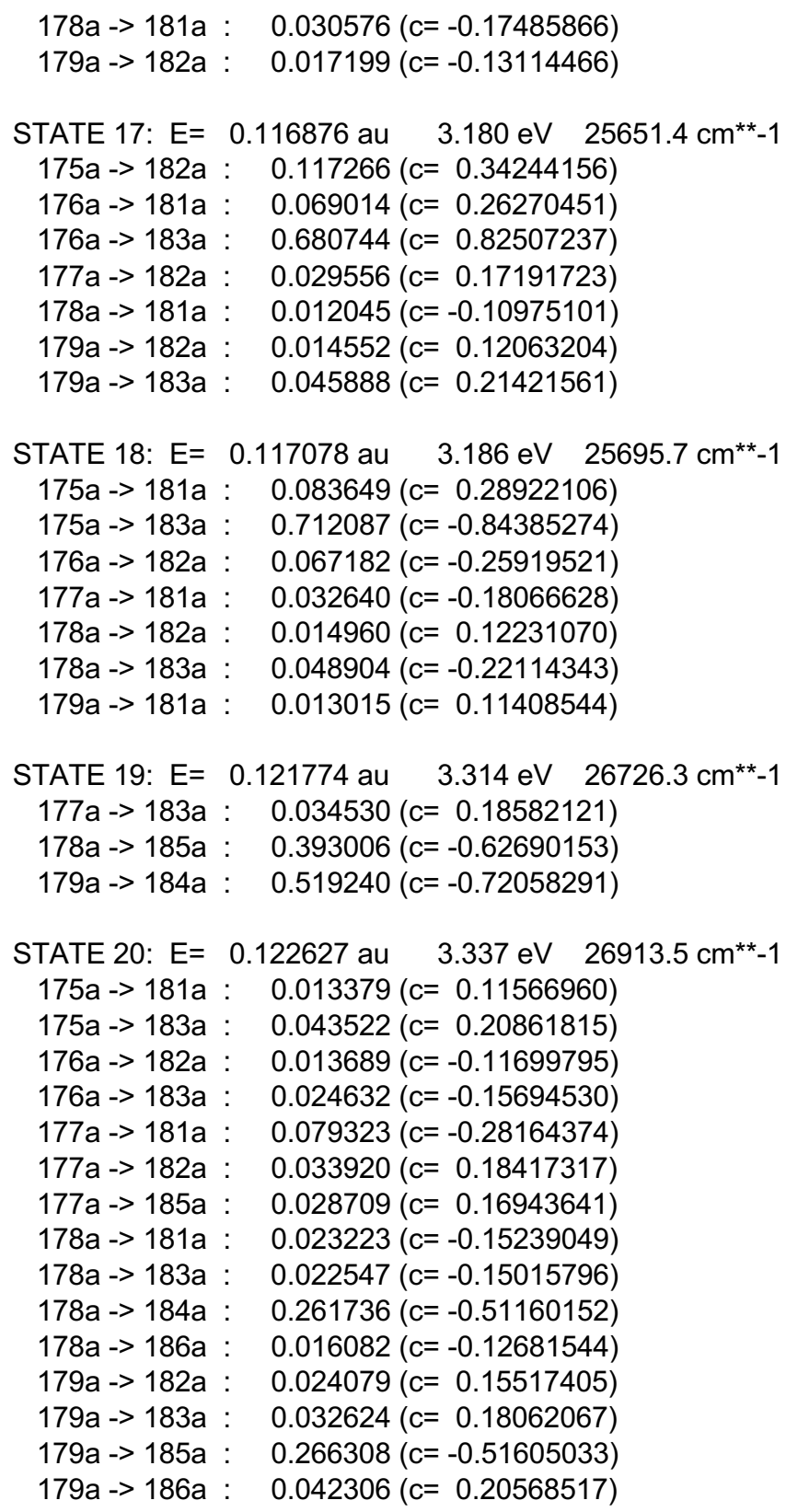


Table S5. Selected Spin-Forbidden Transitions (Singlet Ground to Triplet Excited) from TD-DFT of Bi(bzq) $)_{3}(1)$

\begin{tabular}{cccc} 
State & Transition & Energy (eV) & \multicolumn{2}{c}{ Predicted Wavelength (nm) } \\
\hline 1 & HOMO to LUMO & 2.397 & 517 \\
2 & HOMO to LUMO+1 & 2.399 & 517 \\
\hline 3 & HOMO to LUMO +2 & 2.443 & 508 \\
\hline
\end{tabular}

Transitions with energy difference of less than $0.025 \mathrm{eV}$ are grouped together. 
Table S6. Calculated orbital energies of $\mathrm{Bi}(\mathrm{bzq})_{3}(1)$ around the HOMO and LUMOTriplet Multiplicity

\begin{tabular}{|c|c|c|c|}
\hline Energy (eV) & $\begin{array}{c}\text { Electron } \\
\text { Occupation }\end{array}$ & $\mathrm{Bi} \%$ & $\begin{array}{c}\text { Ligand } \\
\%\end{array}$ \\
\hline-1.2234 & 0 & $4.7 \%$ & $95.3 \%$ \\
\hline-2.0437 & 0 & $2.8 \%$ & $97.2 \%$ \\
\hline-2.0984 & 0 & $3.0 \%$ & $97.0 \%$ \\
-2.1113 & 0 & $3.3 \%$ & $96.7 \%$ \\
\hline-2.4839 & 0 & $1.6 \%$ & $98.4 \%$ \\
\hline-2.5131 & 0 & $1.9 \%$ & $98.1 \%$ \\
-2.5188 & 1 & $2.6 \%$ & $97.4 \%$ \\
\hline-4.2527 & 1 & $32.3 \%$ & $67.7 \%$ \\
\hline-5.4876 & 2 & $1.7 \%$ & $98.3 \%$ \\
\hline-5.5021 & 2 & $1.8 \%$ & $98.2 \%$ \\
-5.5197 & 2 & $1.7 \%$ & $98.3 \%$ \\
\hline-5.8955 & 2 & $0.6 \%$ & $99.4 \%$ \\
\hline-5.9293 & 2 & $0.5 \%$ & $99.5 \%$ \\
\hline-5.9511 & 2 & $0.7 \%$ & $99.3 \%$ \\
\hline
\end{tabular}

Orbital energies within $k_{b} T$ at room temperature $(0.025 \mathrm{eV})$ are grouped together to indicate degeneracy. The percent bismuth and ligand character are given for each orbital.

Based on the higher energy of the bismuth-based orbital in comparison to the HOMO of the singlet state computation (Table S1). The singlet state computations were utilized in the analysis of the behavior of 1 . 


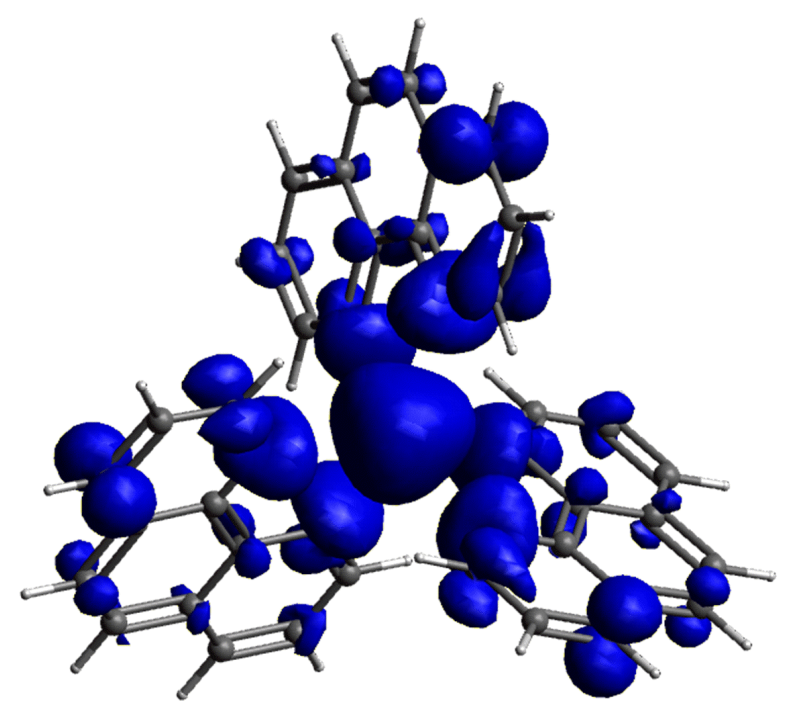

Figure S3. Spin-Density Plot of $\mathrm{Bi}(\mathrm{bzq})_{3}(1)$ with the Triplet Multiplicity

Spin density was calculated through DFT and was plotted on Avogadro. The ISO value is 0.002 .

The total sum of unpaired spin is 2.0000 , the spin on bismuth is 0.32 . 
Table S7. Calculated orbital energies of $\mathrm{Bi}(\mathrm{bzq})_{2} \mathrm{Br}(2)$ around the HOMO and LUMO

\begin{tabular}{|c|c|c|c|}
\hline Energy (eV) & $\begin{array}{c}\text { Electron } \\
\text { Occupation }\end{array}$ & $\mathrm{Bi} \%$ & $\begin{array}{c}\text { Ligand } \\
\%\end{array}$ \\
\hline-0.29932 & 0 & $62.4 \%$ & $37.6 \%$ \\
-0.64681 & 0 & $17.3 \%$ & $82.7 \%$ \\
-0.7483 & 0 & $6.2 \%$ & $93.8 \%$ \\
-1.19075 & 0 & $64.7 \%$ & $35.3 \%$ \\
-1.41361 & 0 & $4.4 \%$ & $95.6 \%$ \\
-1.54749 & 0 & $5.4 \%$ & $94.6 \%$ \\
-2.29634 & 0 & $2.3 \%$ & $97.7 \%$ \\
-2.45987 & 0 & $1.9 \%$ & $98.1 \%$ \\
-2.78749 & 0 & $1.9 \%$ & $98.1 \%$ \\
-2.93525 & 0 & $1.6 \%$ & $98.4 \%$ \\
-4.55621 & 2 & $15.6 \%$ & $84.4 \%$ \\
-4.71376 & 2 & $9.0 \%$ & $91.0 \%$ \\
-4.87757 & 2 & $9.8 \%$ & $90.2 \%$ \\
-5.69553 & 2 & $2.8 \%$ & $97.2 \%$ \\
-5.82941 & 2 & $4.9 \%$ & $95.1 \%$ \\
-6.21962 & 2 & $0.5 \%$ & $99.5 \%$ \\
-6.31513 & 2 & $1.0 \%$ & $99.0 \%$ \\
-6.59894 & 2 & $36.5 \%$ & $63.5 \%$ \\
-6.66425 & 2 & $21.8 \%$ & $78.2 \%$ \\
-6.97364 & 2 & $13.2 \%$ & $86.8 \%$ \\
\hline
\end{tabular}

The percent bismuth and ligand character are given for each orbital. No orbitals in this range are within $k_{b} T$ at room temperature $(0.025 \mathrm{eV})$, indicating a lack of degeneracy. 
Table S8. Calculated orbital energies of Bi(4-phen $)_{3}$ around the HOMO and LUMO

\begin{tabular}{|c|c|c|c|}
\hline Energy (eV) & $\begin{array}{c}\text { Electron } \\
\text { Occupation }\end{array}$ & $\mathrm{Bi} \%$ & $\begin{array}{c}\text { Ligand } \\
\%\end{array}$ \\
\hline-2.0044 & 0 & $2.2 \%$ & $97.8 \%$ \\
-2.0056 & 0 & $2.1 \%$ & $97.9 \%$ \\
\hline-2.0508 & 0 & $1.4 \%$ & $98.6 \%$ \\
\hline-2.0781 & 0 & $6.8 \%$ & $93.2 \%$ \\
-2.0789 & 0 & $6.8 \%$ & $93.2 \%$ \\
\hline-5.3430 & 2 & $3.5 \%$ & $96.5 \%$ \\
\hline-5.3928 & 2 & $1.1 \%$ & $98.9 \%$ \\
-5.3944 & 2 & $1.4 \%$ & $98.6 \%$ \\
\hline-5.6996 & 2 & $20.1 \%$ & $79.9 \%$ \\
-5.7001 & 2 & $2.4 \%$ & $97.6 \%$ \\
\hline
\end{tabular}

Orbital energies within $k_{b} T$ at room temperature $(0.025 \mathrm{eV})$ are grouped together to indicate degeneracy. The percent bismuth and ligand character are given for each orbital. 
Table S9. Cartesian coordinates for the optimized geometry of Bi(bzq) 3 (1) (Ångströms)Singlet

\begin{tabular}{|c|c|c|c|}
\hline$B$ & 11.694878 & 8.880353 & 17.685446 \\
\hline $\mathrm{N}$ & 14.436461 & 9.007613 & 18.48435 \\
\hline $\mathrm{C}$ & 13.458785 & 7.651333 & 20.242003 \\
\hline $\mathrm{C}$ & 12.130206 & 7.817958 & 19.734644 \\
\hline $\mathrm{C}$ & 11.075143 & 7.226829 & 20.44160 \\
\hline $\mathrm{C}$ & 14.625369 & 8.250100 & 19.59662 \\
\hline $\mathrm{C}$ & 13.685343 & 6.877132 & 21.43892 \\
\hline $\mathrm{C}$ & 11.287043 & 6.474705 & 21.62388 \\
\hline $\mathrm{C}$ & 15.477703 & 9.569353 & 17.87294 \\
\hline $\mathrm{H}$ & 15.254613 & 10.172219 & 16.97929 \\
\hline $\mathrm{C}$ & 16.810579 & 9.424608 & 18.32464 \\
\hline $\mathrm{H}$ & 17.633452 & 9.910944 & 17.78765 \\
\hline $\mathrm{C}$ & 15.944696 & 8.046428 & 20.13567 \\
\hline $\mathrm{C}$ & 16.113735 & 7.252140 & 21.32172 \\
\hline $\mathrm{H}$ & 17.126057 & 7.104701 & 21.72067 \\
\hline $\mathrm{C}$ & 12.577139 & 6.296836 & 22.11341 \\
\hline $\mathrm{C}$ & 15.022616 & 6.696513 & 21.94502 \\
\hline $\mathrm{H}$ & 15.154048 & 6.095931 & 22.85537 \\
\hline $\mathrm{C}$ & 17.036464 & 8.656595 & 19. \\
\hline $\mathrm{H}$ & 18.052707 & 8.516507 & 19.85622 \\
\hline $\mathrm{H}$ & 10.047236 & 7.347656 & 20.074 \\
\hline $\mathrm{H}$ & 12.756754 & 5.711404 & 23.02506 \\
\hline $\mathrm{H}$ & 10.431098 & 6.030806 & 22.14946 \\
\hline $\mathrm{C}$ & 9.370960 & 8.631849 & 17.9243 \\
\hline $\mathrm{C}$ & 8.680771 & 7.448693 & 17.50703 \\
\hline $\mathrm{C}$ & 8.607939 & 9.711776 & 18.38699 \\
\hline $\mathrm{C}$ & 7.192731 & 9.665891 & 18.44538 \\
\hline $\mathrm{C}$ & 6.515938 & 8.524459 & 18.0275 \\
\hline $\mathrm{C}$ & 7.238975 & 7.397018 & 17.552 \\
\hline $\mathrm{H}$ & 9.116347 & 10.626754 & 18.71888 \\
\hline $\mathrm{H}$ & 6.633135 & 10.534251 & 18.8178 \\
\hline $\mathrm{H}$ & 5.419259 & 8.476954 & 18.06160 \\
\hline $\mathrm{C}$ & 9.382553 & 6.256898 & 17.03466 \\
\hline $\mathrm{C}$ & 6.540482 & 6.212503 & 17.121 \\
\hline $\mathrm{C}$ & 8.646658 & 5.093066 & 16.61426 \\
\hline $\mathrm{C}$ & 7.210171 & 5.102777 & 16.66525 \\
\hline $\mathrm{H}$ & 6.662552 & 4.208191 & 16.34041 \\
\hline $\mathrm{H}$ & 5.442873 & 6.214749 & 17.1655 \\
\hline $\mathrm{N}$ & 10.741214 & 6.269038 & 17.00896 \\
\hline $\mathrm{C}$ & 9.386770 & 3.966579 & 16.16812 \\
\hline $\mathrm{C}$ & 10.778157 & 4.011077 & $16.1507 \varepsilon$ \\
\hline $\mathrm{C}$ & 11.411629 & 5.198834 & 16.58614 \\
\hline $\mathrm{H}$ & 12.509880 & 5.274102 & 16.59308 \\
\hline $\mathrm{H}$ & 8.847828 & 3.066338 & 15.84317 \\
\hline $\mathrm{H}$ & 11.375806 & 3.155305 & 15.81541 \\
\hline $\mathrm{C}$ & 11.673051 & 11.012451 & 18.67099 \\
\hline $\mathrm{C}$ & 12.015328 & 11.148710 & 20.02254 \\
\hline $\mathrm{C}$ & 12.134353 & 12.415250 & 20.64703 \\
\hline $\mathrm{H}$ & 12.197790 & 10.248720 & 20.62461 \\
\hline $\mathrm{C}$ & 11.917736 & 13.576294 & 19.91181 \\
\hline $\mathrm{H}$ & 12.401654 & 12.476841 & 21.71036 \\
\hline $\mathrm{C}$ & 11.564014 & 13.497998 & 18.53756 \\
\hline $\mathrm{H}$ & 12.013118 & 14.564829 & 20.38065 \\
\hline $\mathrm{C}$ & 11.432252 & 12.203253 & 17.91322 \\
\hline $\mathrm{C}$ & 11.040281 & 12.171480 & 16.50558 \\
\hline $\mathrm{C}$ & 11.337474 & 14.699209 & 17.77418 \\
\hline $\mathrm{C}$ & 10.984835 & 14.657727 & 16.4469 \\
\hline $\mathrm{C}$ & 10.821917 & 13.395233 & 15.77926 \\
\hline $\mathrm{H}$ & 11.453787 & 15.665212 & 18.28413 \\
\hline $\mathrm{H}$ & 10.816013 & 15.582607 & 15.87965 \\
\hline $\mathrm{C}$ & 10.440983 & 13.297065 & 14.4150 \\
\hline $\mathrm{C}$ & 10.286928 & 12.045869 & 13.82426 \\
\hline $\mathrm{H}$ & 10.267961 & 14.216759 & 13.83999 \\
\hline $\mathrm{C}$ & 10.520502 & 10.900311 & 14.62097 \\
\hline $\mathrm{H}$ & 9.989649 & 11.939347 & 12.77436 \\
\hline $\mathrm{N}$ & 10.878954 & 10.964669 & 15.9019 \\
\hline $\mathrm{H}$ & 10.404784 & 9.892052 & 14.19450 \\
\hline
\end{tabular}




\section{Table S10. Cartesian coordinates for the optimized geometry of Bi(bza $)_{3}(1)$} (Ångströms)-Triplet

\begin{tabular}{|c|c|c|c|}
\hline $\mathrm{Bi}$ & 11.325670 & 9.071496 & 18.256112 \\
\hline $\mathrm{N}$ & 13.936961 & 8.733076 & 18.271814 \\
\hline $\mathrm{C}$ & 13.312124 & 7.644882 & 20.353059 \\
\hline $\mathrm{C}$ & 11.930231 & 7.953016 & 20.178591 \\
\hline $\mathrm{C}$ & 10.987696 & 7.532355 & 21.115256 \\
\hline $\mathrm{C}$ & 14.321330 & 8.047697 & 19.390628 \\
\hline $\mathrm{C}$ & 13.725046 & 6.906565 & 21.523556 \\
\hline $\mathrm{C}$ & 11.393110 & 6.810202 & 22.271410 \\
\hline $\mathrm{C}$ & 14.850015 & 9.131287 & 17.364882 \\
\hline $\mathrm{H}$ & 14.466632 & 9.682180 & 16.494638 \\
\hline $\mathrm{C}$ & 16.220341 & 8.866154 & 17.512844 \\
\hline $\mathrm{H}$ & 16.928715 & 9.212101 & 16.751101 \\
\hline $\mathrm{C}$ & 15.704081 & 7.726911 & 19.61 \\
\hline $\mathrm{C}$ & 16.067632 & 6.995378 & 20.79 \\
\hline $\mathrm{H}$ & 17.127336 & 6.757786 & 20.95 \\
\hline $\mathrm{C}$ & 12.737985 & 6.505267 & 22.4 \\
\hline $\mathrm{C}$ & 15.115300 & 6.599416 & 21.71 \\
\hline $\mathrm{H}$ & 15.411106 & 6.041613 & 22.61 \\
\hline $\mathrm{C}$ & 16.653327 & 8.155161 & 18.6 \\
\hline $\mathrm{H}$ & 17.716081 & 7.925962 & 18.7 \\
\hline $\mathrm{H}$ & 9.921156 & 7.752071 & 20.967182 \\
\hline $\mathrm{H}$ & 13.056041 & 5.944713 & 23.35 \\
\hline $\mathrm{H}$ & 10.642459 & 6.493241 & 23.00 \\
\hline $\mathrm{C}$ & 9.040529 & 8.686102 & 18.277984 \\
\hline $\mathrm{C}$ & 8.556616 & 7.503463 & 17.649578 \\
\hline $\mathrm{C}$ & 8.160928 & 9.584209 & 18.879177 \\
\hline $\mathrm{C}$ & 6.762772 & 9.320525 & 18.89 \\
\hline $\mathrm{C}$ & 6.268258 & 8.159800 & 18.305057 \\
\hline $\mathrm{C}$ & 7.139577 & 7.225539 & 17.674793 \\
\hline $\mathrm{H}$ & 8.539453 & 10.502197 & 19.349947 \\
\hline $\mathrm{H}$ & 6.077663 & 10.035245 & 19.365041 \\
\hline $\mathrm{H}$ & 5.190519 & 7.949006 & 18.316211 \\
\hline $\mathrm{C}$ & 9.439920 & 6.558347 & 16.981106 \\
\hline $\mathrm{C}$ & 6.639885 & 6.026335 & 17.064631 \\
\hline $\mathrm{C}$ & 8.902084 & 5.366006 & 16.385558 \\
\hline $\mathrm{C}$ & 7.486998 & 5.128178 & 16.448080 \\
\hline $\mathrm{H}$ & 7.089957 & 4.212503 & 15.990554 \\
\hline $\mathrm{H}$ & 5.559320 & 5.833680 & 17.097078 \\
\hline $\mathrm{N}$ & 10.776805 & 6.820790 & 16.930538 \\
\hline $\mathrm{C}$ & 9.812309 & 4.469004 & 15.755829 \\
\hline $\mathrm{C}$ & 11.180738 & 4.766662 & 15.736614 \\
\hline $\mathrm{C}$ & 11.622145 & 5.958710 & 16.337029 \\
\hline $\mathrm{H}$ & 12.686405 & 6.232744 & 16.344045 \\
\hline $\mathrm{H}$ & 9.429072 & 3.550495 & 15.293527 \\
\hline $\mathrm{H}$ & 11.904206 & 4.092885 & 15.262762 \\
\hline $\mathrm{C}$ & 11.352871 & 11.235841 & 19.065846 \\
\hline $\mathrm{C}$ & 11.409494 & 11.495097 & 20.434086 \\
\hline $\mathrm{C}$ & 11.458926 & 12.834151 & 20.911857 \\
\hline $\mathrm{H}$ & 11.421317 & 10.666496 & 21.155805 \\
\hline $\mathrm{C}$ & 11.471934 & 13.894576 & 20.010724 \\
\hline $\mathrm{H}$ & 11.490273 & 13.026475 & 21.992095 \\
\hline $\mathrm{C}$ & 11.432994 & 13.665402 & 18.606285 \\
\hline $\mathrm{H}$ & 11.516716 & 14.930080 & 20.373863 \\
\hline $\mathrm{C}$ & 11.369332 & 12.305024 & 18.123678 \\
\hline $\mathrm{C}$ & 11.337826 & 12.077447 & 16.686855 \\
\hline $\mathrm{C}$ & 11.453343 & 14.751342 & 17.667824 \\
\hline $\mathrm{C}$ & 11.417436 & 14.525871 & 16.307168 \\
\hline $\mathrm{C}$ & 11.360483 & 13.191186 & 15.777768 \\
\hline $\mathrm{H}$ & 11.496115 & 15.777553 & 18.056351 \\
\hline $\mathrm{H}$ & 11.431608 & 15.367752 & 15.602564 \\
\hline $\mathrm{C}$ & 11.321001 & 12.911233 & 14.382724 \\
\hline $\mathrm{C}$ & 11.263908 & 11.582539 & 13.939574 \\
\hline $\mathrm{H}$ & 11.333800 & 13.743005 & 13.666954 \\
\hline $\mathrm{C}$ & 11.254119 & 10.552558 & 14.895554 \\
\hline $\mathrm{H}$ & 11.226749 & 11.338495 & 12.871459 \\
\hline $\mathrm{N}$ & 11.290722 & 10.798208 & 16.217588 \\
\hline $\mathrm{H}$ & 11.210617 & 9.496367 & 14.594223 \\
\hline
\end{tabular}


Table S11. Cartesian coordinates for the optimized geometry of $\mathrm{Bi}(\mathrm{bzq})_{2} \mathrm{Br}(2)$ (Ångströms)

\begin{tabular}{|c|c|c|c|}
\hline $\mathrm{Bi}$ & 2.068248 & 4.539967 & 10.869953 \\
\hline $\mathrm{Br}$ & 4.998618 & 5.024900 & 11.181605 \\
\hline $\mathrm{N}$ & 1.409004 & 2.465662 & 9.482390 \\
\hline 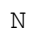 & 1.917696 & 6.017170 & 12.882340 \\
\hline $\mathrm{C}$ & 0.984377 & 2.424012 & 8.215936 \\
\hline $\mathrm{C}$ & 2.871124 & 2.711945 & 13.449116 \\
\hline $\mathrm{C}$ & 2.374872 & 2.673162 & 12.144911 \\
\hline $\mathrm{C}$ & 3.048700 & 1.519780 & 14.194507 \\
\hline$\checkmark$ & 1.524023 & 7.740976 & 15.043979 \\
\hline C & 2.257751 & 0.198315 & 12.300467 \\
\hline 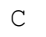 & 2.795597 & 7.558511 & 14.505084 \\
\hline C & 1.584380 & 1.315500 & 10.191340 \\
\hline $\mathrm{C}$ & 0.690124 & 1.211167 & 7.555780 \\
\hline $\mathrm{C}$ & 2.954193 & 6.678454 & 13.411825 \\
\hline C & 2.745281 & 0.281174 & 13.631904 \\
\hline $\mathrm{C}$ & 2.069326 & 1.414576 & 11.555094 \\
\hline C & -1.271863 & 3.913034 & 11.086930 \\
\hline $\mathrm{C}$ & 0.656039 & 6.175377 & 13.380776 \\
\hline $\mathrm{C}$ & -2.586612 & 4.065208 & 11.600623 \\
\hline C & 0.413586 & 7.050745 & 14.491589 \\
\hline $\mathrm{C}$ & -0.183756 & 4.585782 & 11.6532 \\
\hline C & -0.433225 & 5.445745 & 12.760018 \\
\hline $\mathrm{C}$ & -0.929357 & 7.190750 & 14.989814 \\
\hline $\mathrm{C}$ & -2.828837 & 4.904584 & 12.686458 \\
\hline C & -1.758940 & 5.617956 & 13.291 \\
\hline $\mathrm{C}$ & -1.969294 & 6.500374 & 14.411268 \\
\hline $\mathrm{C}$ & 1.307385 & 0.035986 & 9.600591 \\
\hline $\mathrm{C}$ & 0.852146 & 0.016702 & 8.256858 \\
\hline $\mathrm{C}$ & 1.958552 & -1.068306 & 11.681227 \\
\hline $\mathrm{C}$ & 1.503436 & -1.153387 & 10.385771 \\
\hline $\mathrm{H}$ & 0.873795 & 3.388401 & 7.700010 \\
\hline $\mathrm{H}$ & 3.146591 & 3.670703 & 13.904759 \\
\hline $\mathrm{H}$ & 3.437572 & 1.575473 & 15.219318 \\
\hline $\mathrm{H}$ & 1.366366 & 8.416734 & 15.895212 \\
\hline $\mathrm{H}$ & 3.670583 & 8.079128 & 14.909921 \\
\hline $\mathrm{H}$ & 3.931926 & 6.478927 & 12.937601 \\
\hline $\mathrm{H}$ & -1.123027 & 3.241986 & 10.229374 \\
\hline $\mathrm{H}$ & -3.844894 & 5.024809 & 13.084696 \\
\hline $\mathrm{H}$ & 0.344760 & 1.222595 & 6.515964 \\
\hline $\mathrm{H}$ & -2.989884 & 6.615643 & 14.800285 \\
\hline $\mathrm{H}$ & -1.106326 & 7.861281 & 15.84059 \\
\hline $\mathrm{H}$ & 2.108889 & -1.982070 & 12.271367 \\
\hline $\mathrm{H}$ & 1.287121 & -2.128032 & 9.930000 \\
\hline $\mathrm{H}$ & -3.418300 & 3.516432 & 11.138710 \\
\hline $\mathrm{H}$ & 2.889053 & -0.643114 & 14.20631 \\
\hline & 0.633870 & -0.946793 & 7.77771 \\
\hline
\end{tabular}




\section{Table S12. Cartesian coordinates for the optimized geometry of Bi(phenanthrene) $)_{3}$} (Ångströms)

\begin{tabular}{|c|c|c|c|}
\hline $\mathrm{Bi}$ & 11.468048 & 8.976508 & 18.084220 \\
\hline C & 14.605791 & 9.584601 & 18.874971 \\
\hline $\mathrm{C}$ & 13.569553 & 7.842940 & 20.405608 \\
\hline $\mathrm{C}$ & 12.192649 & 8.146453 & 20.123746 \\
\hline $\mathrm{C}$ & 11.190110 & 7.630665 & 20.958488 \\
\hline $\mathrm{C}$ & 14.708290 & 8.419761 & 19.686751 \\
\hline C & 13.855032 & 6.880886 & 21.445051 \\
\hline $\mathrm{C}$ & 11.491233 & 6.792179 & 22.059031 \\
\hline $\mathrm{C}$ & 15.692313 & 10.078984 & 18.150869 \\
\hline $\mathrm{H}$ & 15.571091 & 10.985786 & 17.545664 \\
\hline $\mathrm{C}$ & 16.949073 & 9.434790 & 18.224716 \\
\hline $\mathrm{H}$ & 17.800815 & 9.816522 & 17.648225 \\
\hline $\mathrm{C}$ & 16.018021 & 7.838261 & 19.839608 \\
\hline $\mathrm{C}$ & 16.222666 & 6.794164 & 20.808256 \\
\hline $\mathrm{H}$ & 17.229307 & 6.370263 & 20.919703 \\
\hline $\mathrm{C}$ & 12.805389 & 6.394123 & 22.271959 \\
\hline $\mathrm{C}$ & 15.194632 & 6.378609 & 21.616611 \\
\hline $\mathrm{H}$ & 15.368661 & 5.624308 & 22.395574 \\
\hline $\mathrm{C}$ & 17.107657 & 8.344884 & 19.077102 \\
\hline $\mathrm{H}$ & 18.091697 & 7.871241 & 19.192649 \\
\hline $\mathrm{H}$ & 10.136675 & 7.848333 & 20.737835 \\
\hline $\mathrm{H}$ & 13.052055 & 5.683505 & 23.072096 \\
\hline $\mathrm{H}$ & 10.683173 & 6.419732 & 22.701482 \\
\hline $\mathrm{C}$ & 9.242672 & 8.379982 & 18.346599 \\
\hline $\mathrm{C}$ & 8.649339 & 7.249798 & 17.684763 \\
\hline $\mathrm{C}$ & 8.412279 & 9.362003 & 18.906789 \\
\hline $\mathrm{C}$ & 7.001141 & 9.289974 & 18.813835 \\
\hline $\mathrm{C}$ & 6.418691 & 8.268666 & 18.073171 \\
\hline $\mathrm{C}$ & 7.219644 & 7.255932 & 17.477118 \\
\hline $\mathrm{H}$ & 8.860887 & 10.233403 & 19.401799 \\
\hline $\mathrm{H}$ & 6.381113 & 10.067237 & 19.278438 \\
\hline $\mathrm{H}$ & 5.331367 & 8.236756 & 17.922380 \\
\hline $\mathrm{C}$ & 9.401703 & 6.100749 & 17.175355 \\
\hline $\mathrm{C}$ & 6.608484 & 6.263299 & 16.630055 \\
\hline $\mathrm{C}$ & 8.764830 & 5.171624 & 16.276565 \\
\hline $\mathrm{C}$ & 7.357153 & 5.297116 & 16.006264 \\
\hline $\mathrm{H}$ & 6.885508 & 4.574228 & 15.327720 \\
\hline $\mathrm{H}$ & 5.523645 & 6.318060 & 16.468313 \\
\hline $\mathrm{C}$ & 10.736349 & 5.807699 & 17.574215 \\
\hline $\mathrm{C}$ & 9.519669 & 4.103158 & 15.716239 \\
\hline $\mathrm{C}$ & 10.852445 & 3.898594 & 16.064937 \\
\hline $\mathrm{C}$ & 11.453350 & 4.741021 & 17.028923 \\
\hline $\mathrm{H}$ & 12.476380 & 4.544989 & 17.372793 \\
\hline $\mathrm{H}$ & 9.019944 & 3.421039 & 15.015510 \\
\hline $\mathrm{H}$ & 11.418454 & 3.066461 & 15.628223 \\
\hline $\mathrm{C}$ & 11.212708 & 11.172143 & 18.786217 \\
\hline $\mathrm{C}$ & 11.432073 & 11.397866 & 20.153373 \\
\hline $\mathrm{C}$ & 11.647286 & 12.697427 & 20.672589 \\
\hline $\mathrm{H}$ & 11.476031 & 10.544658 & 20.843131 \\
\hline $\mathrm{C}$ & 11.731748 & 13.773977 & 19.798288 \\
\hline $\mathrm{H}$ & 11.799106 & 12.838821 & 21.750263 \\
\hline $\mathrm{C}$ & 11.528062 & 13.593791 & 18.402415 \\
\hline $\mathrm{H}$ & 11.973746 & 14.778786 & 20.169523 \\
\hline $\mathrm{C}$ & 11.174628 & 12.291864 & 17.884732 \\
\hline $\mathrm{C}$ & 10.830523 & 12.200479 & 16.463360 \\
\hline $\mathrm{C}$ & 11.718316 & 14.702596 & 17.502153 \\
\hline $\mathrm{C}$ & 11.570249 & 14.555663 & 16.145970 \\
\hline $\mathrm{C}$ & 11.101610 & 13.314243 & 15.589767 \\
\hline $\mathrm{H}$ & 12.018270 & 15.669340 & 17.928119 \\
\hline $\mathrm{H}$ & 11.761196 & 15.397193 & 15.467155 \\
\hline $\mathrm{C}$ & 10.843606 & 13.195994 & 14.195176 \\
\hline C & 10.276643 & 12.042191 & 13.659705 \\
\hline $\mathrm{H}$ & 11.079496 & 14.049564 & 13.545722 \\
\hline $\mathrm{C}$ & 9.908721 & 10.985935 & 14.525055 \\
\hline $\mathrm{H}$ & 10.078606 & 11.969607 & 12.583123 \\
\hline $\mathrm{C}$ & 10.172878 & 11.074129 & 15.893105 \\
\hline $\mathrm{H}$ & 9.390113 & 10.103641 & 14.130266 \\
\hline $\mathrm{H}$ & 13.681742 & 10.173152 & 18.884729 \\
\hline $\mathrm{H}$ & 11.191069 & 6.368105 & 18.39853 \\
\hline $\mathrm{H}$ & 9.773829 & 10.290705 & 16.54649 \\
\hline
\end{tabular}




\section{$\mathrm{Bi}(\mathrm{bzq})_{3}(1)$ Characterization}

LMBibzq3-d8THF-01092019.10.fid

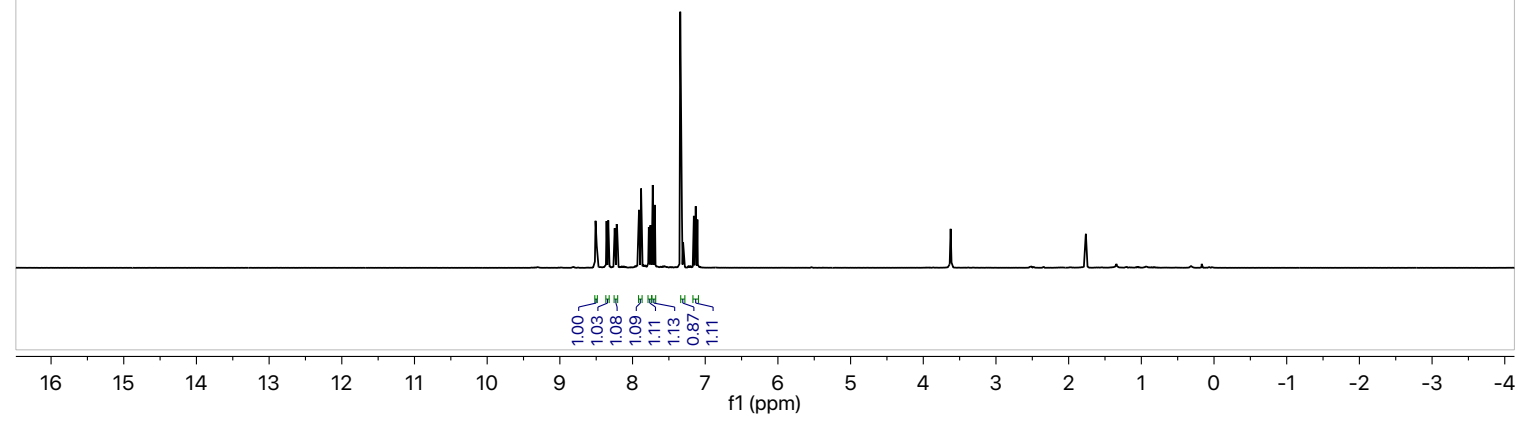

LMBibzq3-d8THF-01092019.10.fid

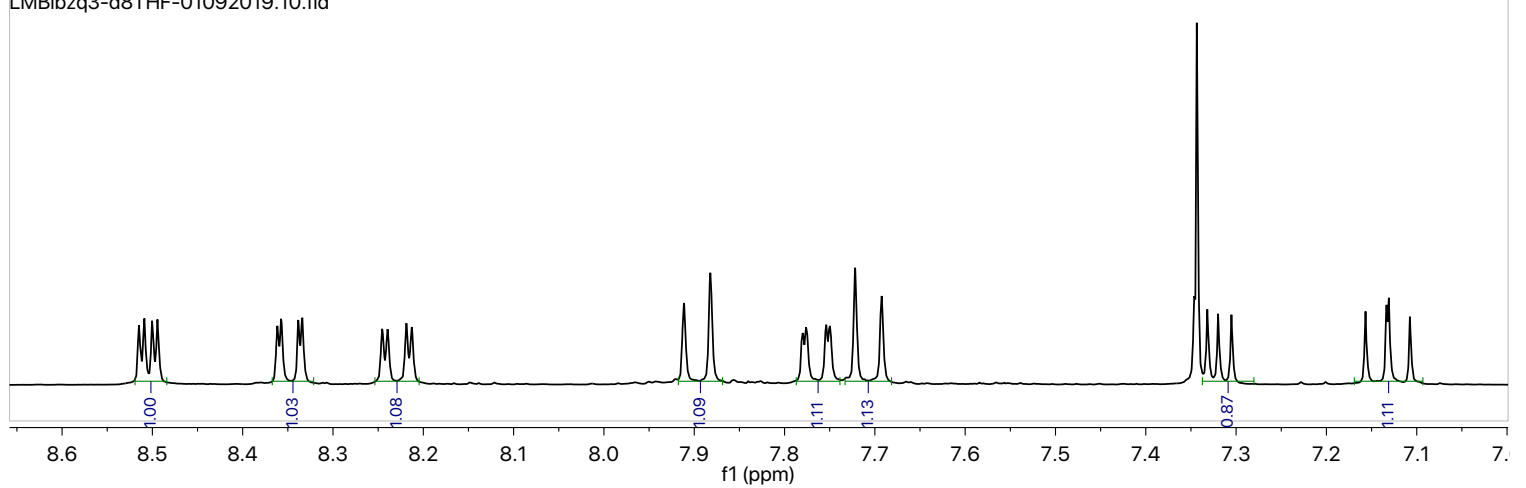

Fig S4. ${ }^{1} \mathrm{H}$ NMR spectrum of $\mathrm{Bi}(\mathrm{bzq})_{3}$ in THF-d8 (1)

Peak at $7.35 \mathrm{ppm}$ is residual benzene from the benzene wash. 

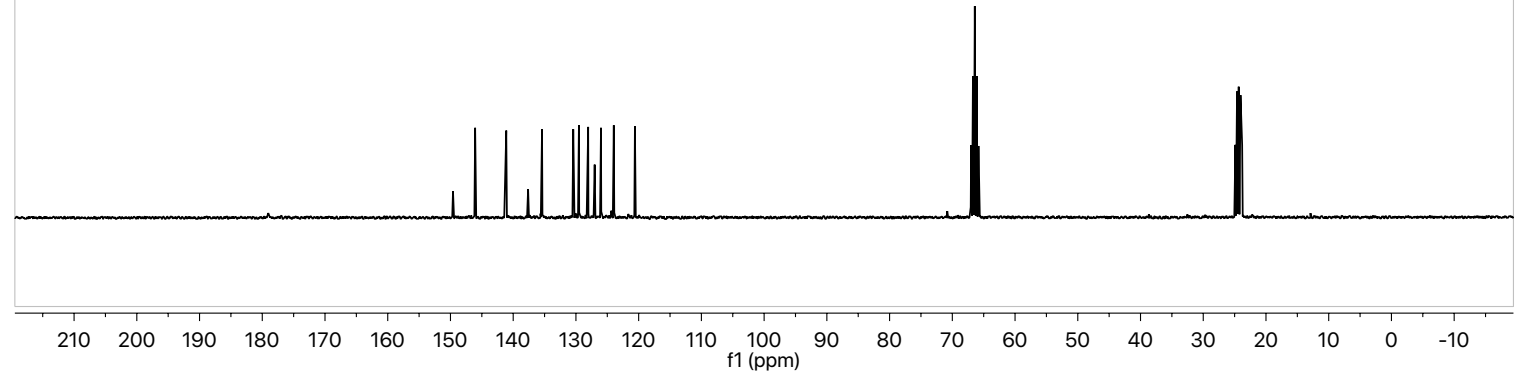

LMBibzq3-d8THF-01092019.11.fid

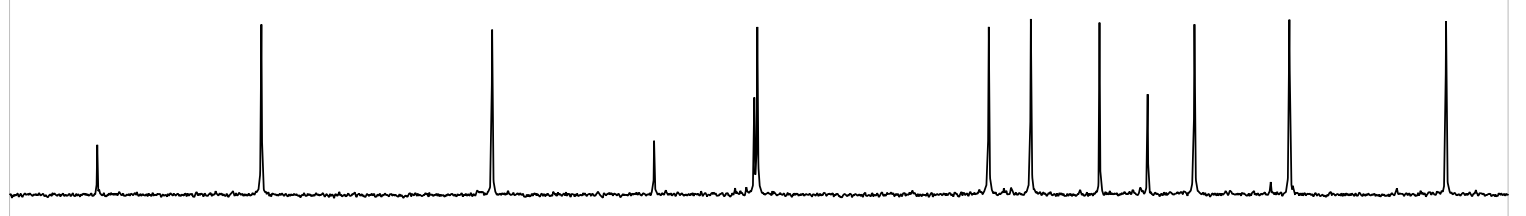

$\begin{array}{lllllllllllllllllllllllllllllllllll}151 & 150 & 149 & 148 & 147 & 146 & 145 & 144 & 143 & 142 & 141 & 140 & 139 & 138 & 137 & 136 & 135 & 134 & 133 & 132 & 131 & 130 & 129 & 128 & 127 & 126 & 125 & 124 & 123 & 122 & 121 & 120\end{array}$

Fig. S5. ${ }^{13} \mathrm{C}$ NMR Spectrum of $\mathrm{Bi}(\mathrm{bzq})_{3}$ in THF-d8 (1) 
SYNAPT-G2\#UCB300

DDJ190111_LMaurer_MarchakLab_LM261B_ESI- $(0.052)$ Is $(1.00,1.00)$ C39H24N3Bi

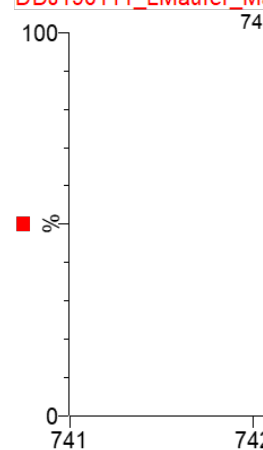

DDJ190111_LMaurer_MarchakLab_LM261B_ESI- 14 (0.294) AM2 (Ar,20000.0,0.00,0.00); ABS; Cm (14:24)

$1007-742.1678$

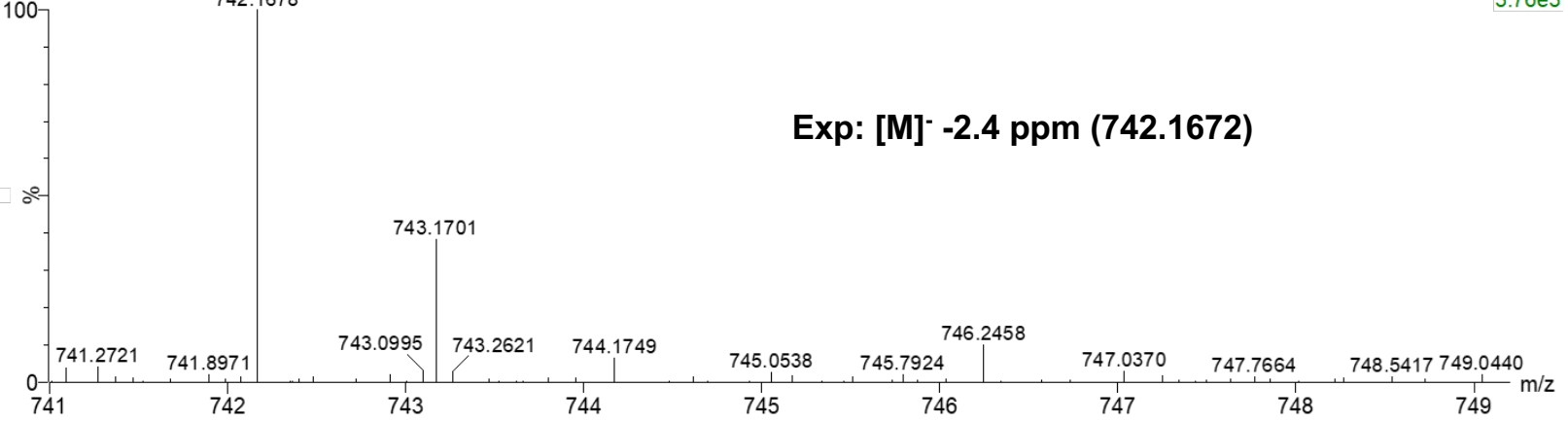

Fig. S6. High Resolution Mass Spectrum of $\mathrm{Bi}(\mathrm{bzq})_{3}(\mathbf{1})$ 


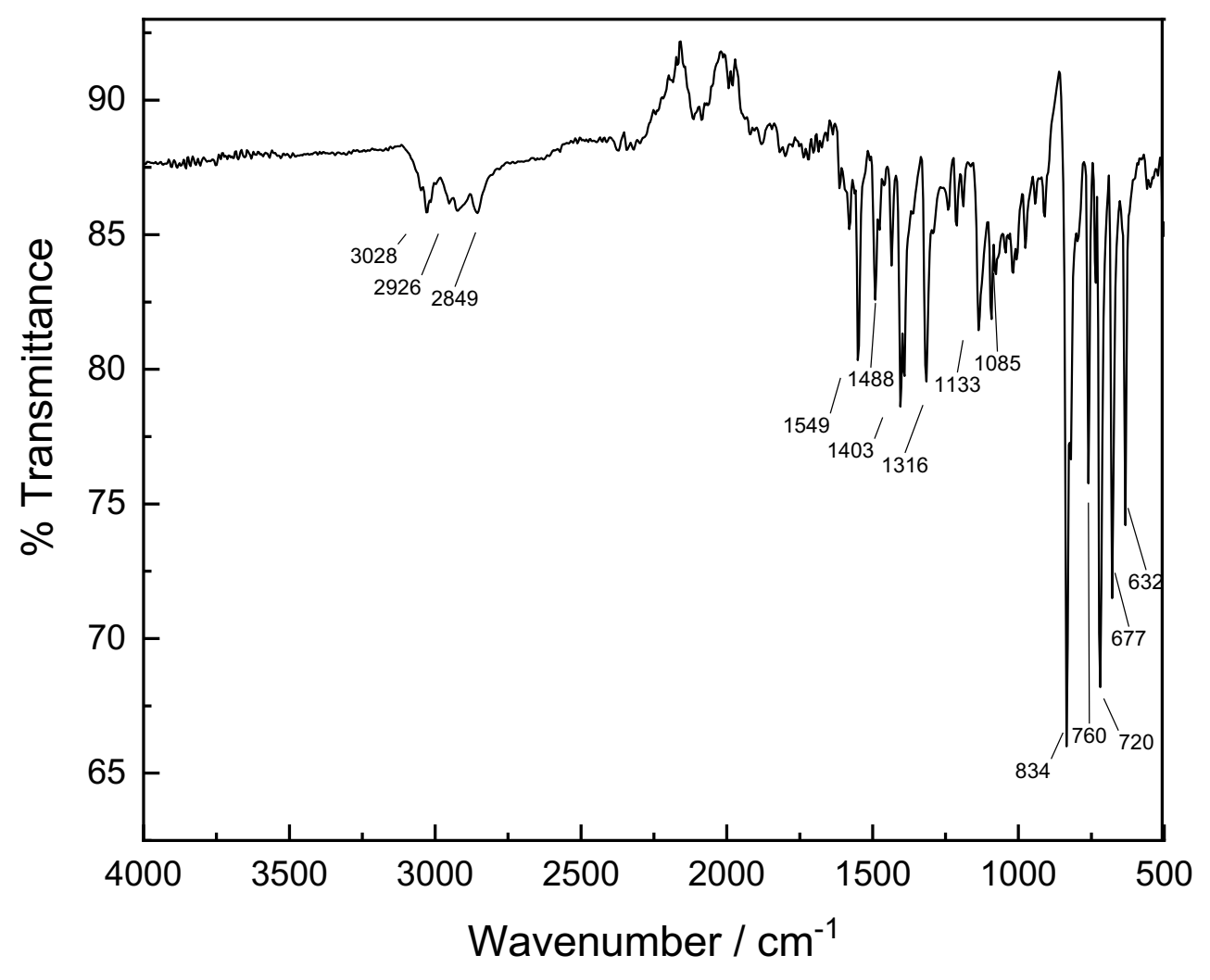

Fig. S7. Infrared Spectrum of Bi(bzq $)_{3}(1)$ 


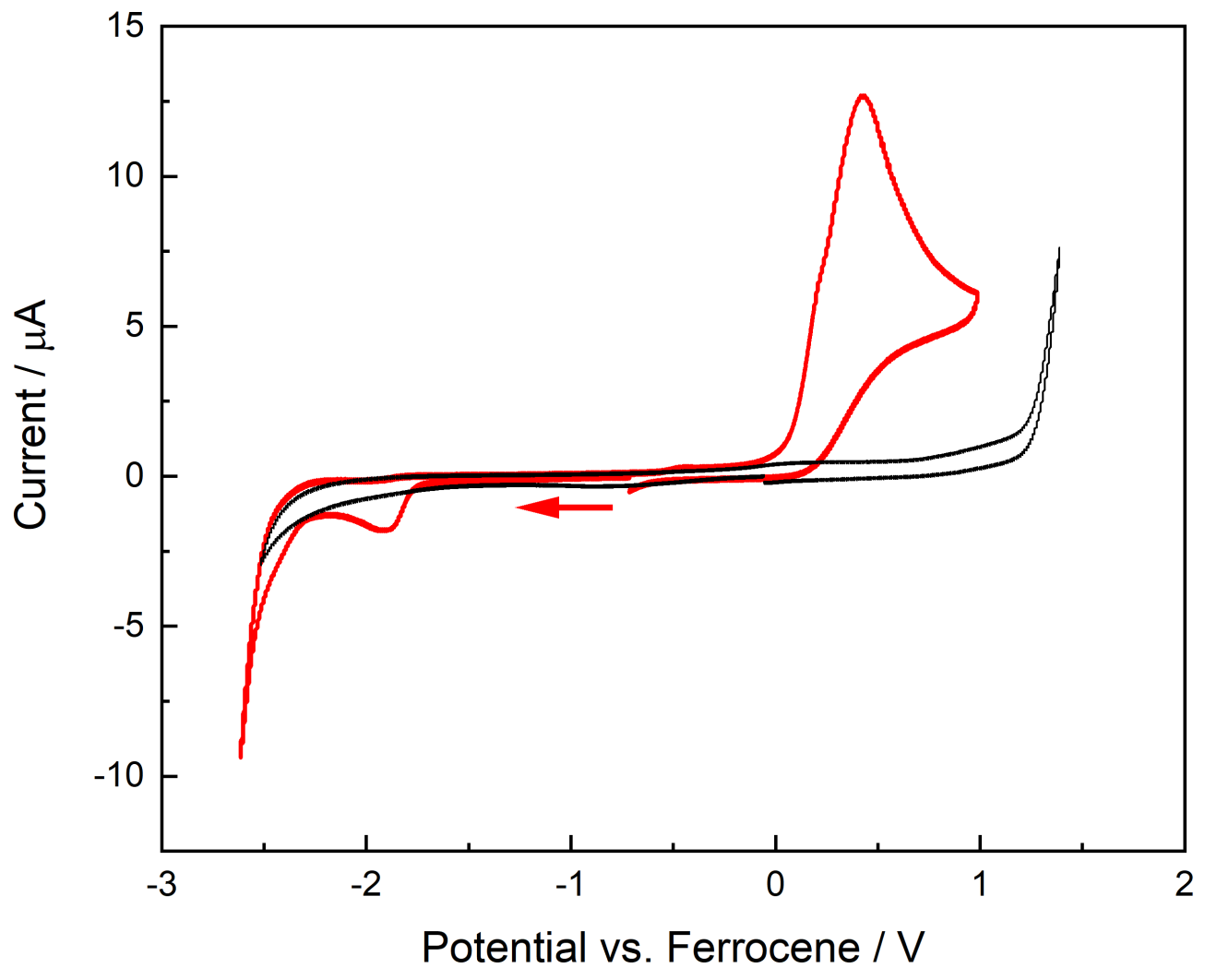

Fig. S8. Cyclic Voltammogram of Bi(bzq) $)_{3}(1)$

Scans were collected for the DCM solvent background (black) and $\mathrm{Bi}(\mathrm{bzq})_{3}$ (red) starting at the open circuit potential. The scans were collected by first scanning in the negative direction. This is a complete cycle. 


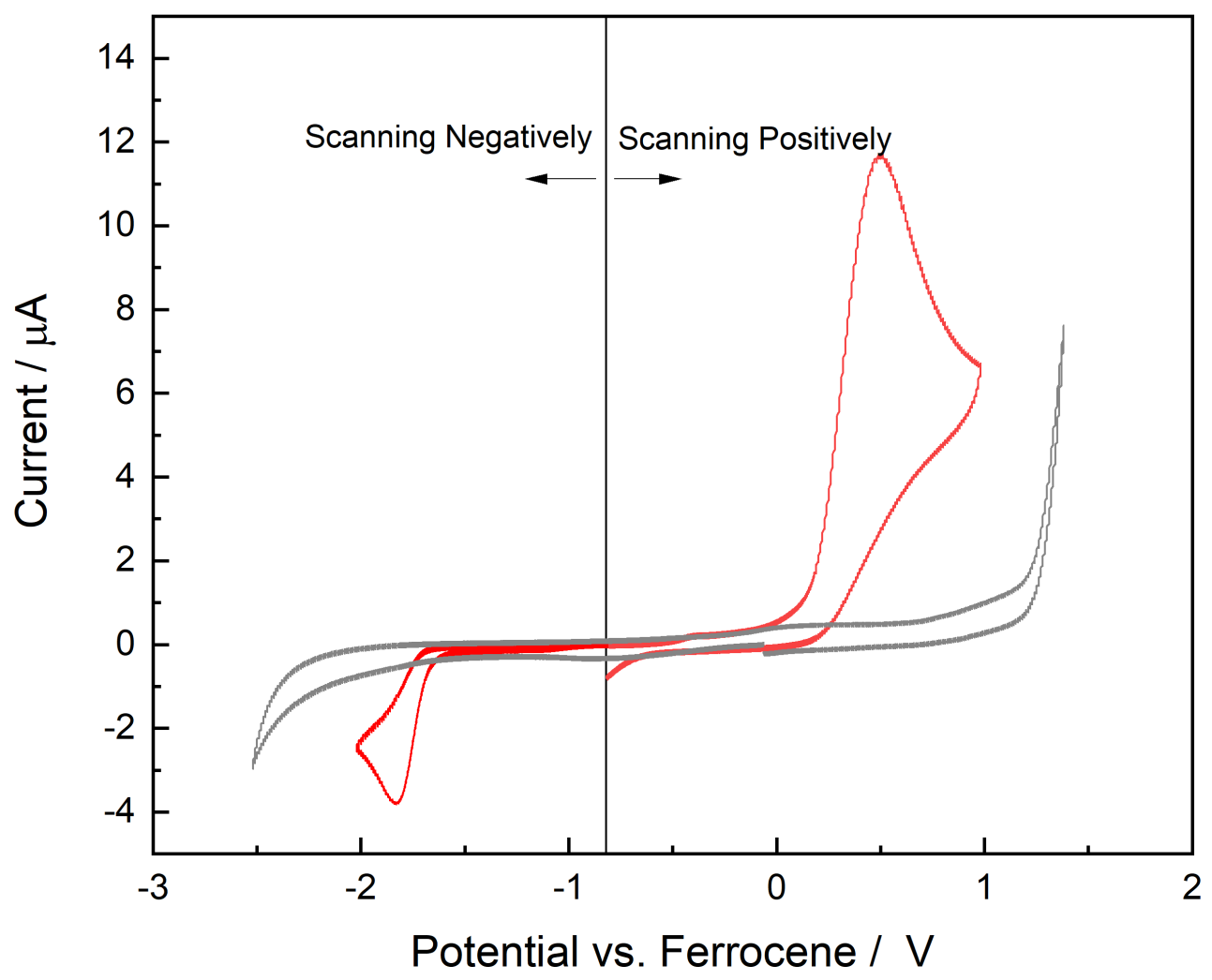

Fig. S9. Cyclic Voltammogram of Bi(bzq $)_{3}(1)$

Scans were collected for DCM (grey) and $\mathrm{Bi}(\mathrm{bzq})_{3}(\mathrm{red})$ starting at the open circuit potential. The scans were taken scanning in the positive direction on the right and stopping at the open circuit potential. Then scans were collected scanning on the negative direction and stopping at the open circuit potential. This was not cycled through, and the vertical line is representing the location of the open circuit potential corrected against ferrocene. 


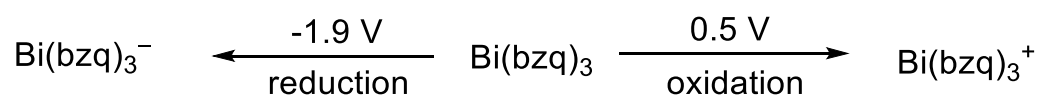

Fig. S10. Ground State Latimer Diagram of Bi(bzq $)_{3}(\mathbf{1})$ 


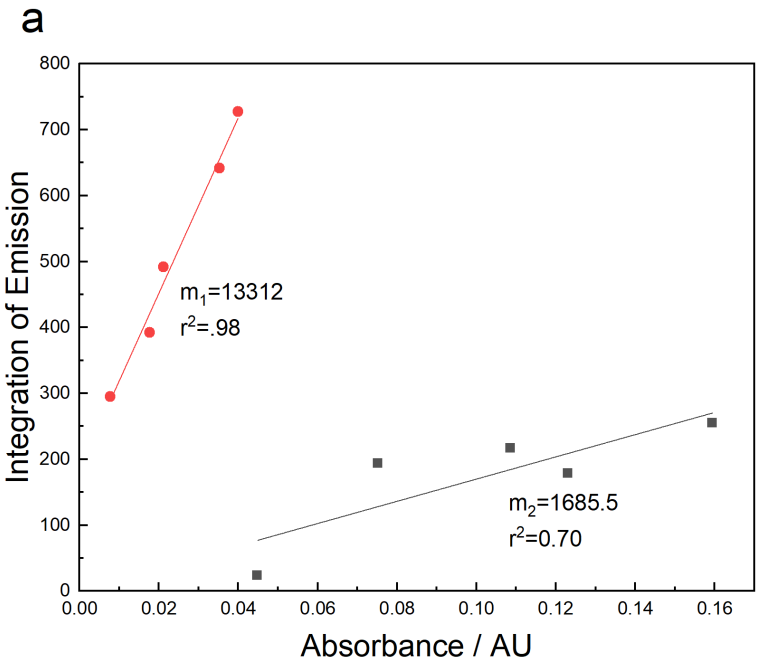

b
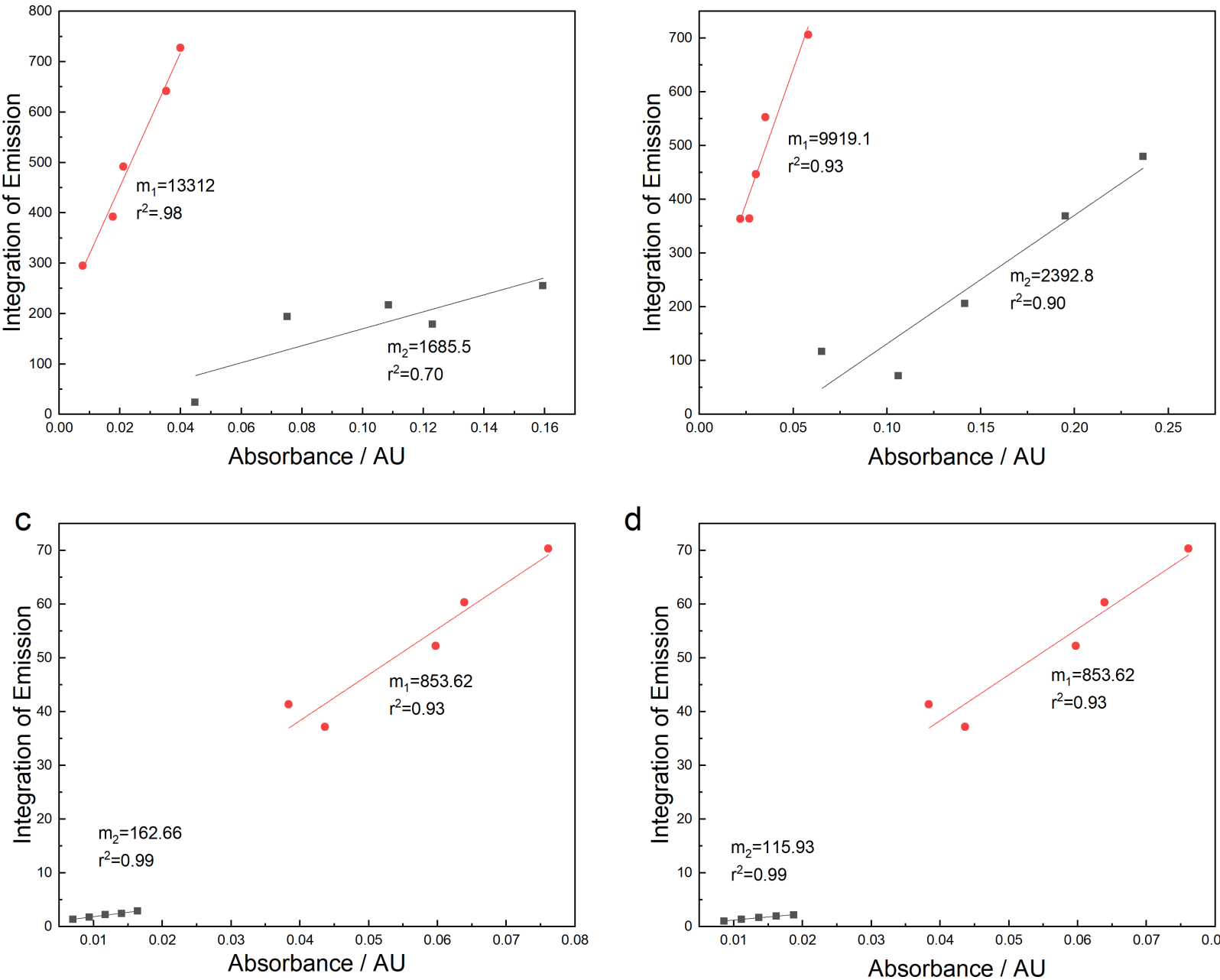

d

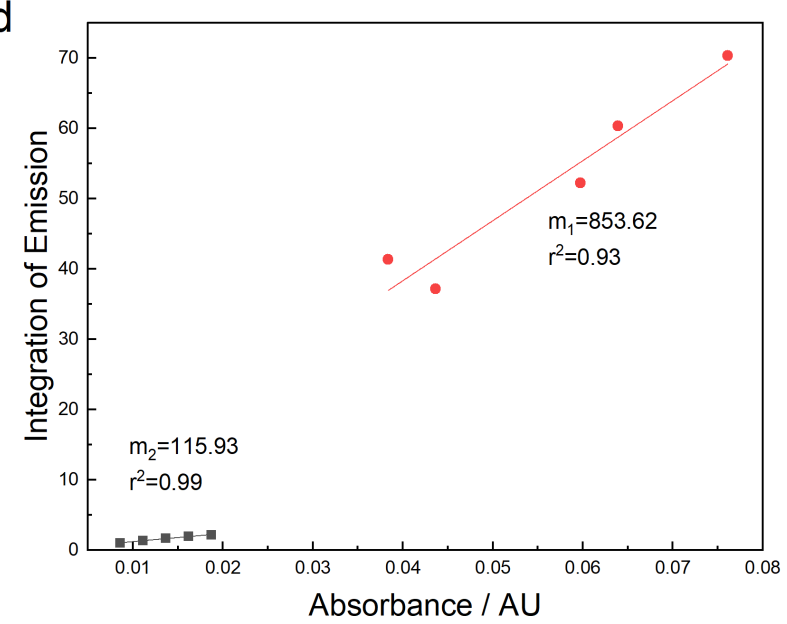

Fig. S11. Quantum Yield Data for Bi(bzq) $)_{3}(1)$

Coumarin 153 is in red and $\mathrm{Bibzq}_{3}(1)$ is in black.

Quantum yields were collected using a comparative method. Several dilutions were prepared in each trial of both 1 and the standard (coumarin 153). Coumarin 153 has a quantum yield of $0.54^{1}$ and these solutions were collected using ethanol as a solvent. Data for 1 was collected in THF.

Data is compiled in Table S7. 
Table S13. Compiled information of quantum yield measurements of 1.

\begin{tabular}{cc}
\hline $\mathbf{B i}(\mathbf{b z q})_{3} \mathbf{5 2 0} \mathbf{n m}$ & $\boldsymbol{\Phi}_{\mathbf{5 2 0}}$ \\
\hline Trial 1 & 0.074 \\
Trial 2 & 0.14 \\
Trial 3 & 0.11 \\
Trial 4 & 0.079 \\
Average & 0.098 \\
Standard Deviation & \pm 0.03 \\
\hline
\end{tabular}

The above table contains the compiled quantum yield values found from the slopes of the graphs from Fig. S7 for the $520 \mathrm{~nm}$ peak. 


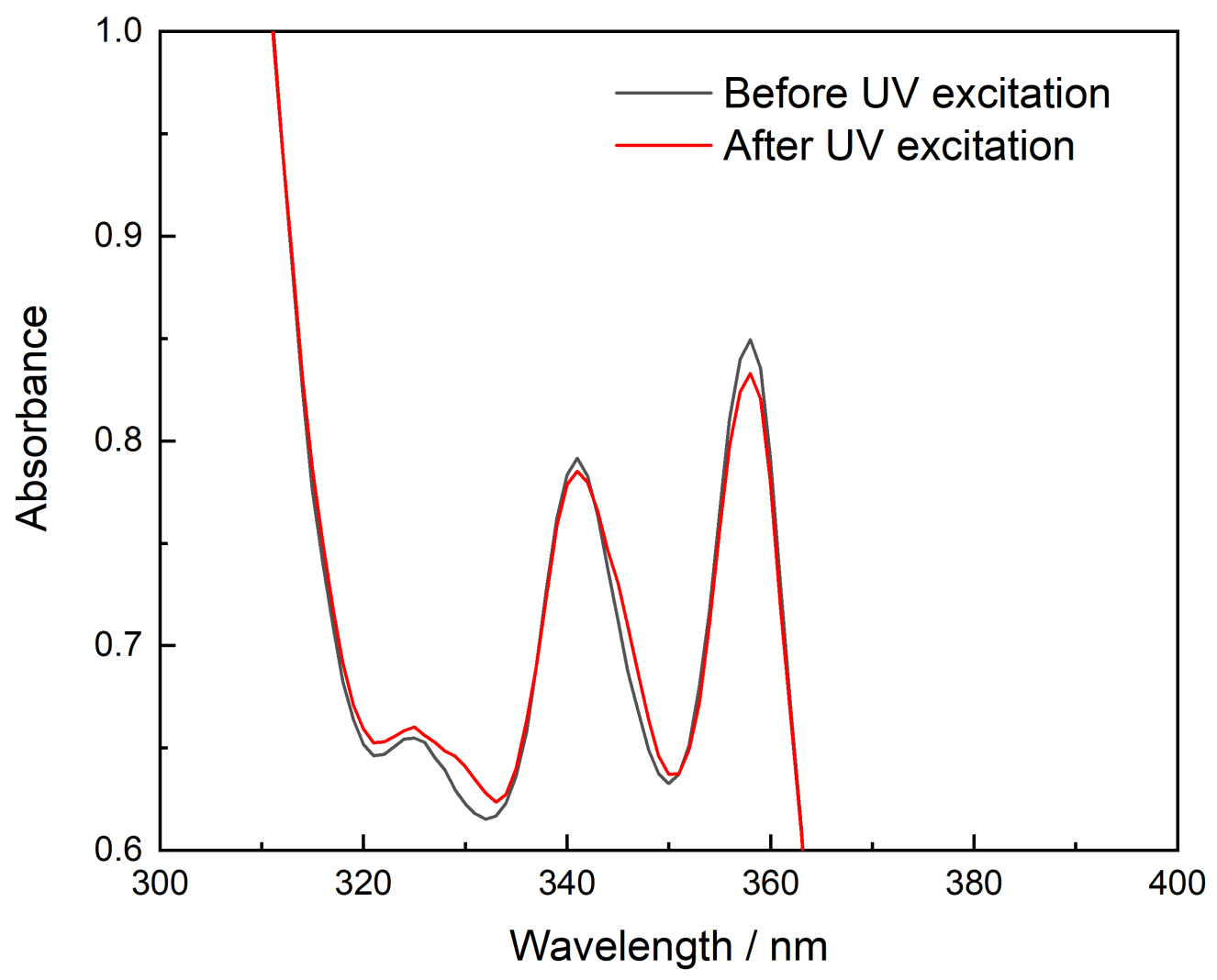

Fig. S12. UV Light Decomposition of Bi(bzq $)_{3}$ (1) through absorption

Absorption was studied in THF before and after UV excitation to show complex degradation. 

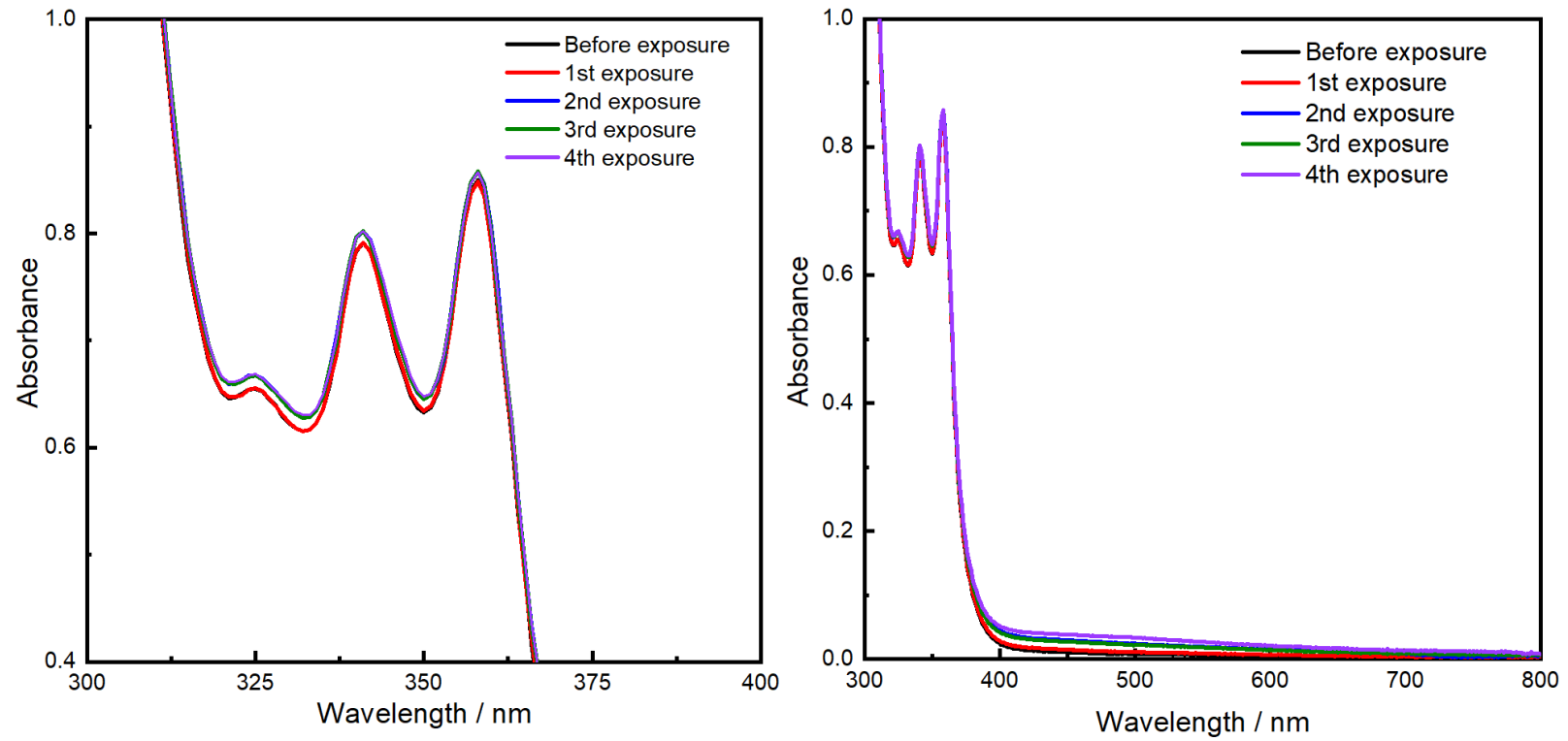

Fig. S13. Stability of $\mathrm{Bi}(\mathrm{bzq})_{3}$ (1) to $450 \mathrm{~nm}$ light through absorption

Absorption spectrum showing the stability of 1 to $450 \mathrm{~nm}$ light after repeated exposures in THF.

Each exposure was collected on a fluorometer with 19 seconds of light exposure. Light exposure through an Horiba Jobin Yvon Fluorolog-3. 


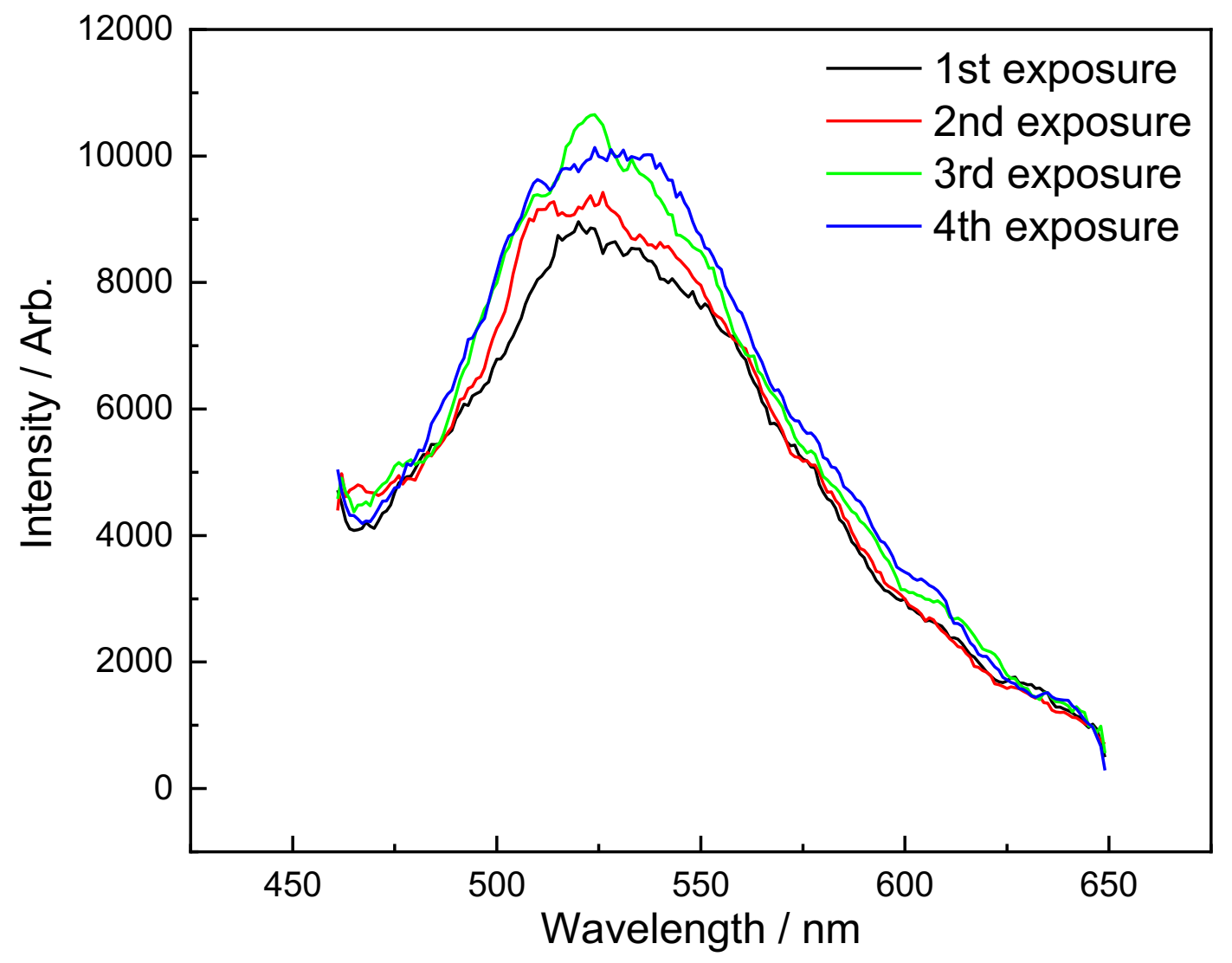

Fig. S14. Stability of Bi(bzq $)_{3}(1)$ to $450 \mathrm{~nm}$ light through emission

Emission spectrum showing the stability of 1 to $450 \mathrm{~nm}$ light after repeated exposures in THF.

Each exposure was through a fluorometer with 19 seconds for each exposure time. Experiments were conducted using an Horiba Jobin Yvon Fluorolog-3. 


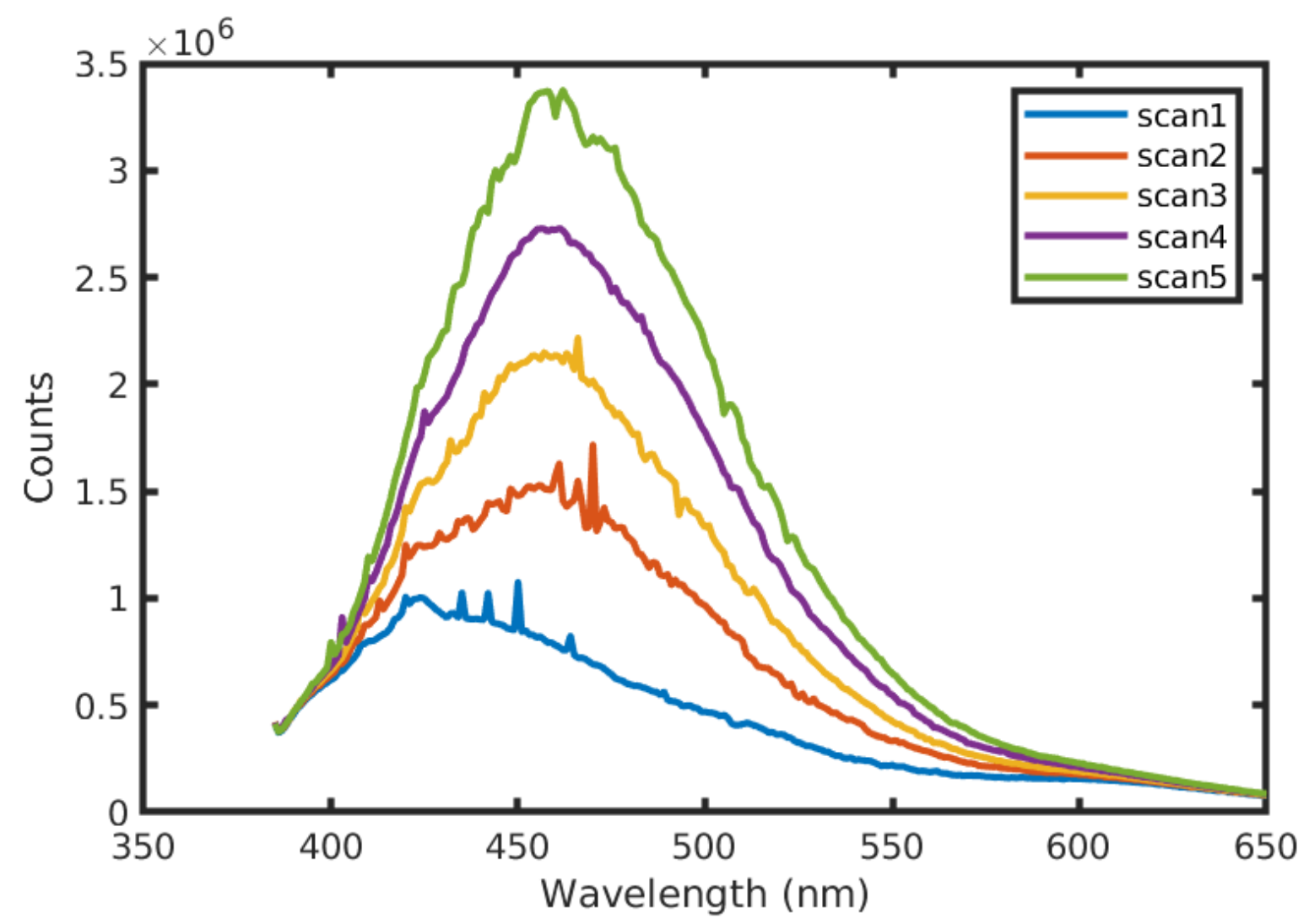

Fig. S15. UV light decomposition of Bi(bzq $)_{3}$ (1) through emission

Breakdown of sample following $375 \mathrm{~nm}$ excitation in toluene, showing emission. 


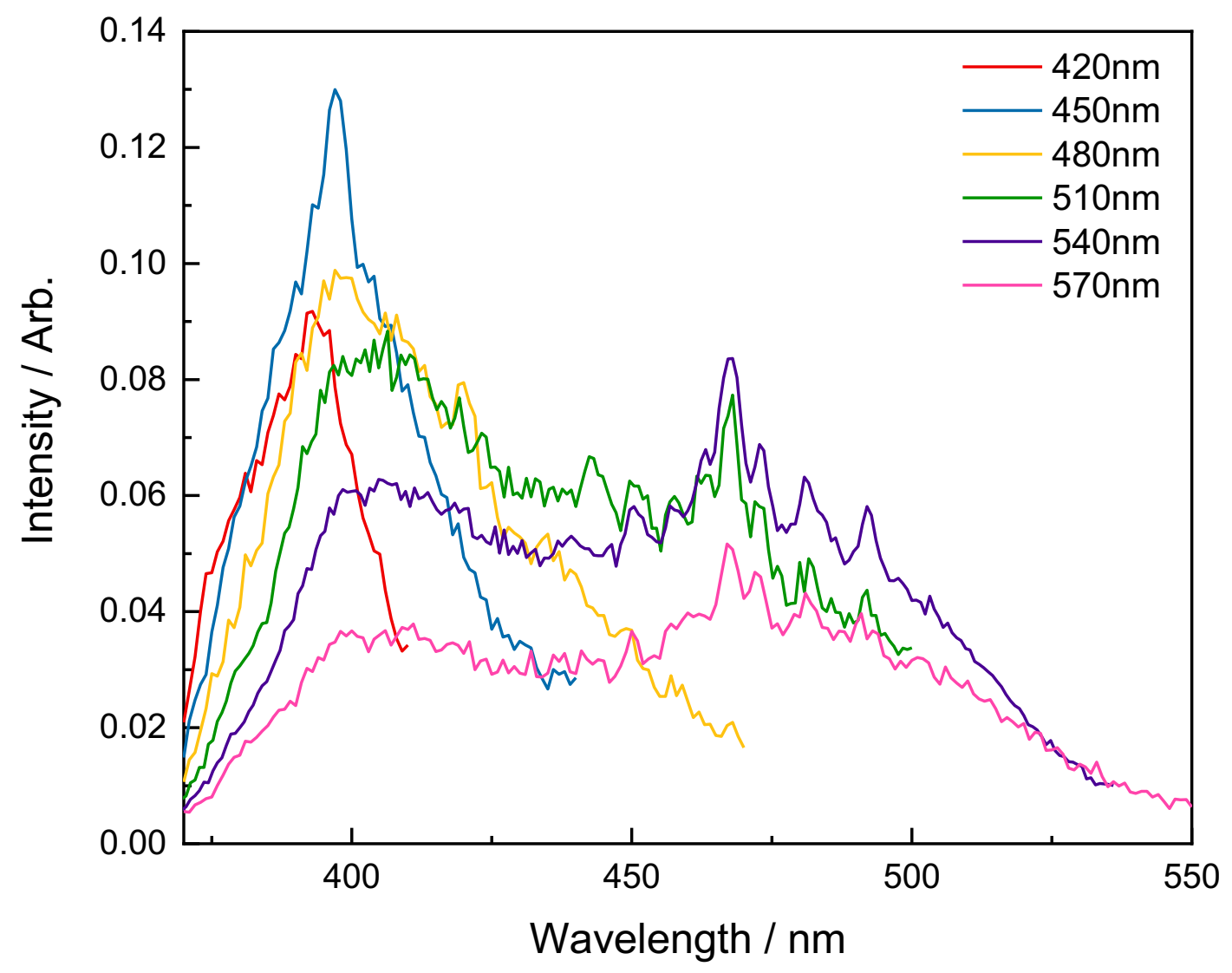

Fig. S16. Excitation Scans of $\mathrm{Bi}(\mathrm{bzq})_{3}(\mathbf{1})$

Excitation Scans of 1 in THF. The legend depicts the wavelength at which the emission was measured. 


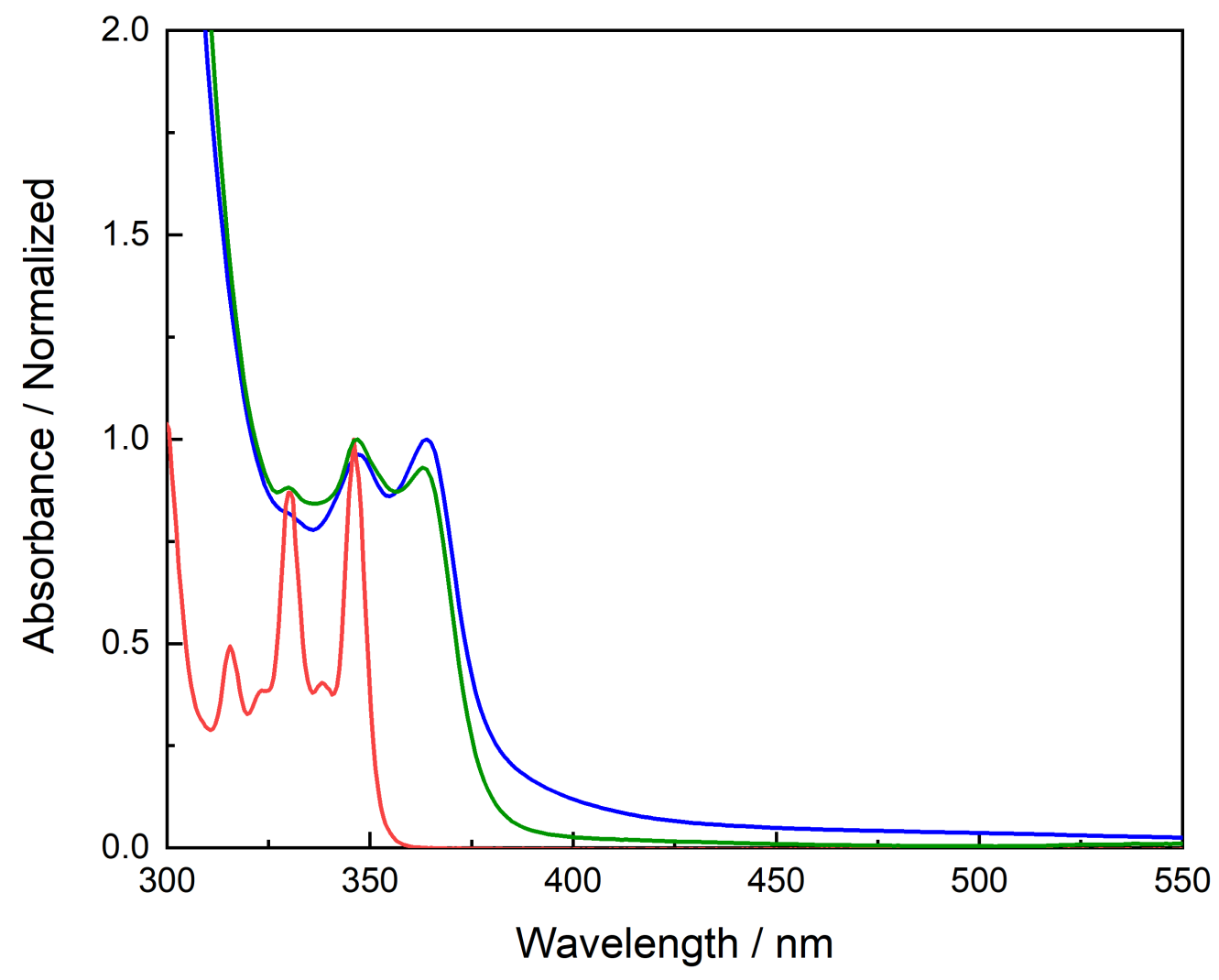

Fig. S17. Normalized Absorbance of $\mathrm{Bi}(\mathrm{bzq})_{3}(\mathbf{1}), \mathrm{Bi}(\mathrm{bzq})_{2} \mathrm{Br}(2)$ and bzq

$\mathrm{Bi}(\mathrm{bzq})_{3}$ is shown above in blue and the bzq ligand is shown in red. $\mathrm{Bi}(\mathrm{bzq})_{2} \mathrm{Br}$ is shown in green and is the average of 12 scans due to the insoluble nature of this compound. The averaging of these scans is to accurately show the baseline of $\mathbf{2}$. 1 and the bzq ligand were collected in THF, while $\mathbf{2}$ is collected in DCM due to limited solubility. 


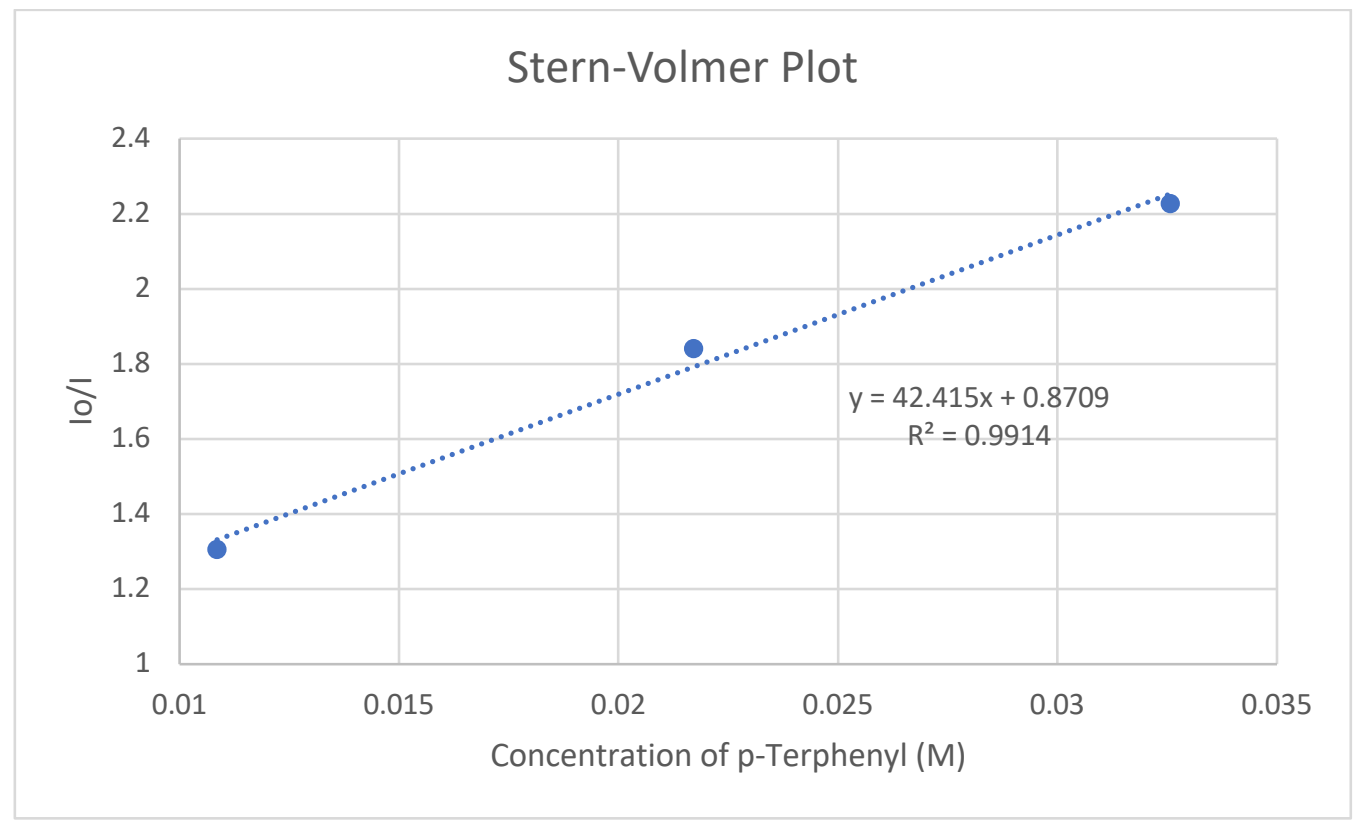

Fig. S18. Stern-Volmer Experiment for Bi(bzq) $)_{3}(1)$

This experiment was conducted in an air and moisture free cuvettes using dried THF. The concentration of $\mathrm{Bi}(\mathrm{bzq})_{3}$ was maintained at $0.00020 \mathrm{M}$ for the entire experiment. The concentration of $\mathrm{p}$-Terphenyl was varied between the three trials, and THF was used to maintain the same overall volume within the cuvette. This experiment was conducted by measuring the steady-state emission of the $525 \mathrm{~nm}$ peak. The emission intensity of the unquenched sample $\left(I_{0}\right)$ was then divided by the emission intensity of the quenched samples (I).

The triplet energy of p-Terphenyl is $2.66 \mathrm{eV}^{2}$.

An average $\mathrm{Kq}$ for these data is $1.0 \times 10^{-6} \mathrm{~s}^{-1} \mathrm{M}^{-1}$ 


\section{$\mathrm{Bi}(\mathrm{bzq}){ }_{2} \mathrm{Br}$ Characterization}

NB_pg146_Bi(bzq)2Br.10.fid

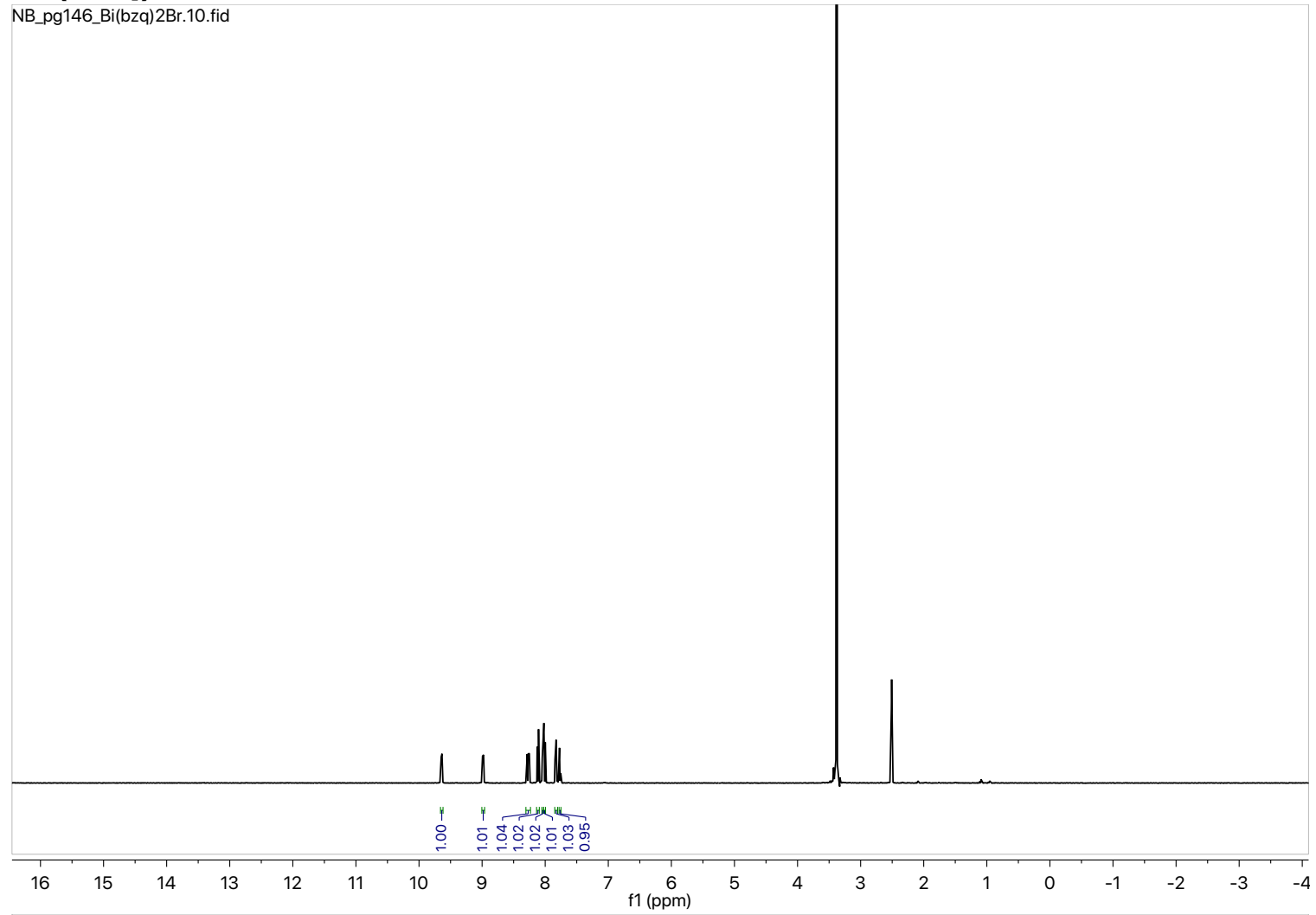

NB_pg146_Bi(bzq)2Br.10.fid

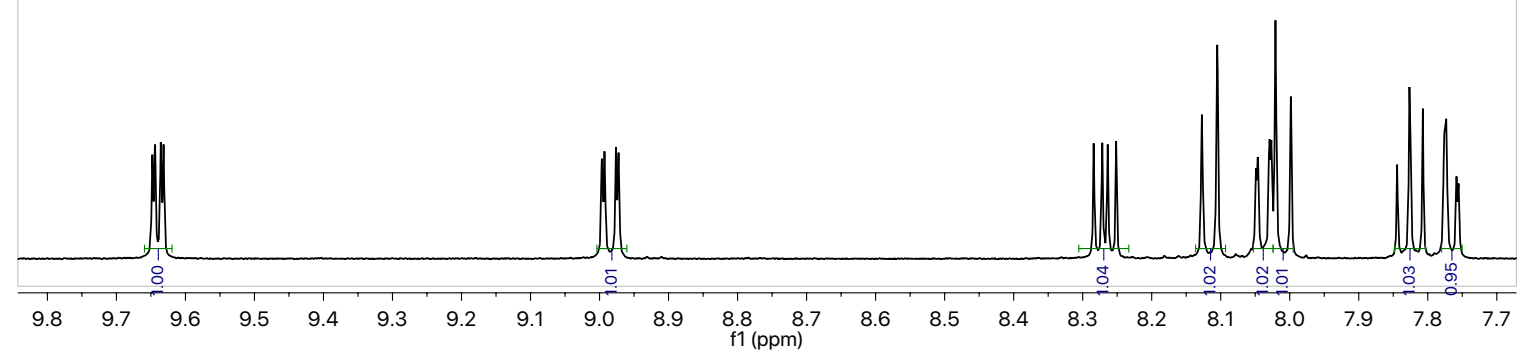

Fig. S19. 1H NMR Spectrum of Bi(bza $)_{2} \mathrm{Br}$ in $\left(\mathrm{CD}_{3}\right)_{2} \mathrm{SO}(2)$ 

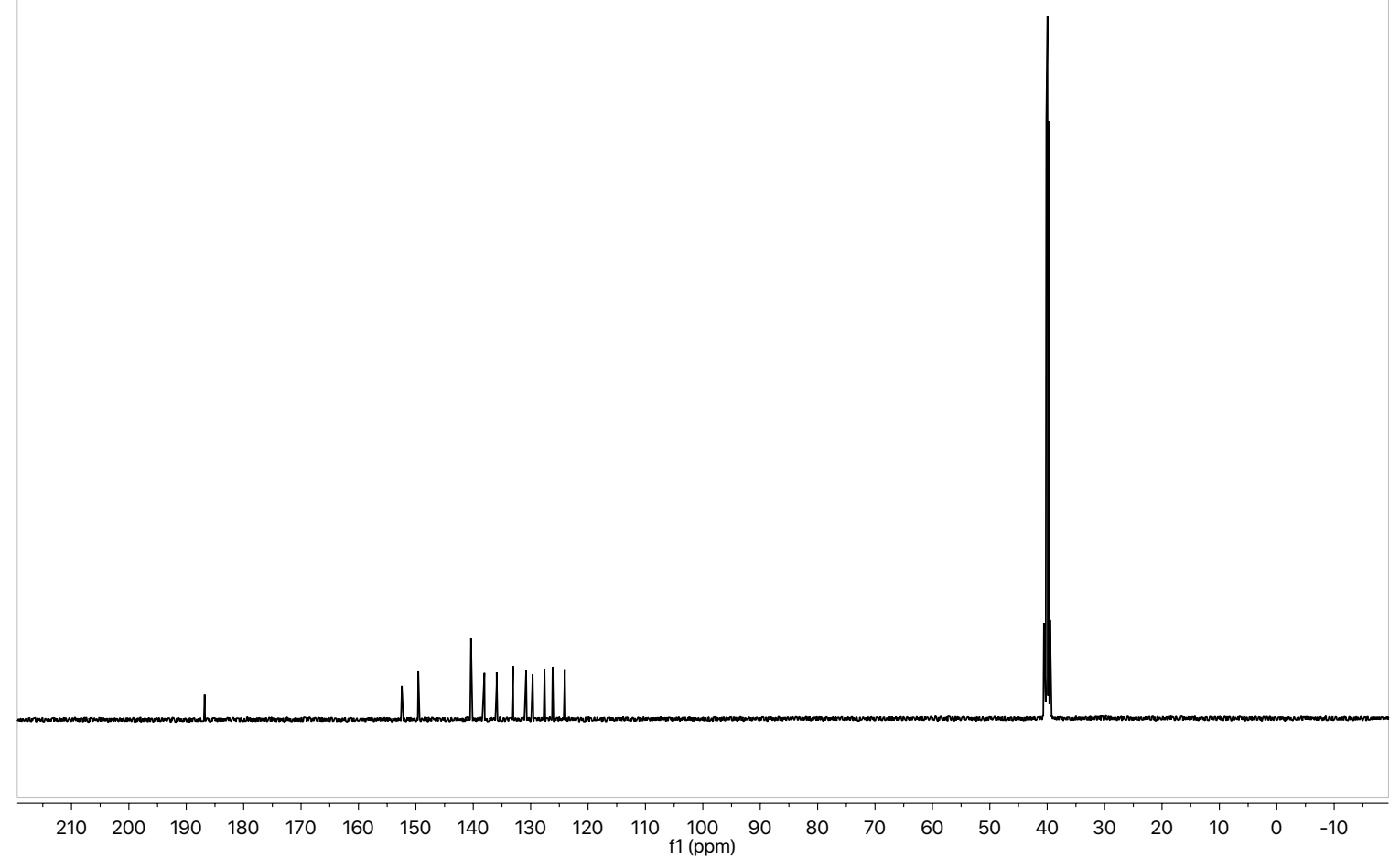

NB_pg146_Bibzq2Br_DMSO.11.fid

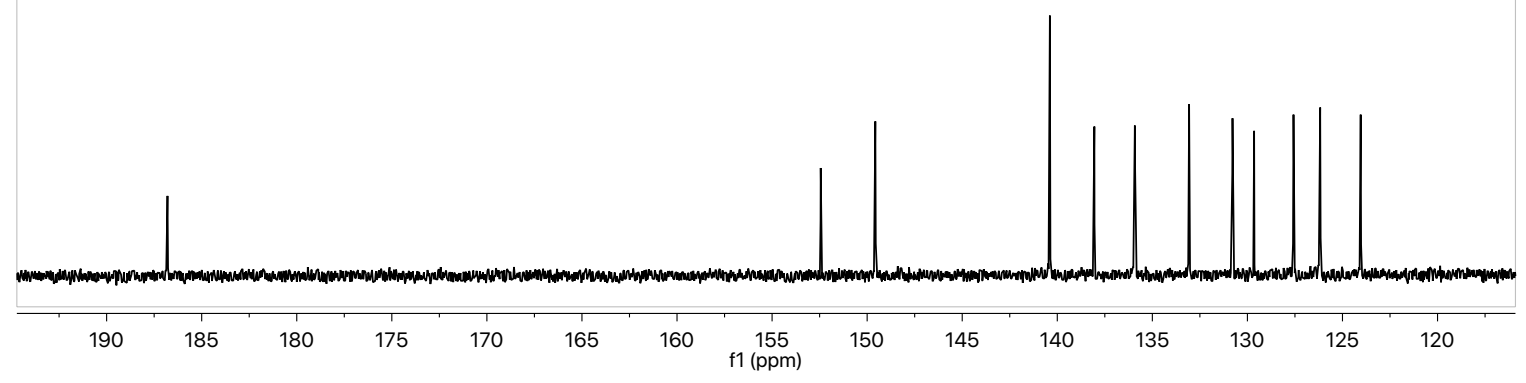

Fig. S20. ${ }^{13} \mathrm{C}$ NMR Spectrum of $\mathrm{Bi}(\mathrm{bzq})_{2} \mathrm{Br}$ in $\left(\mathrm{CD}_{3}\right)_{2} \mathrm{SO}(2)$ 
SYNAPT-G2\#UCB300

DDJ190423_NBrown_MarshakLab_C26H16N2BiBr_ESI+ $(0.052)$ Is $(1.00,1.00)$ C26H16N2Bi

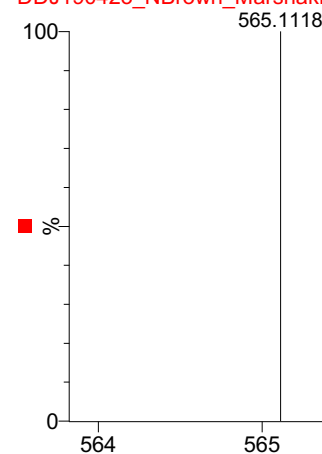

65.1118
DDJ190423_NBrown_MarshakLab_C26H16N2BiBr_ESI+ 21 (0.433) AM2 (Ar, 18000.0,0.00,0.00); ABS; Cm (13:28)

100

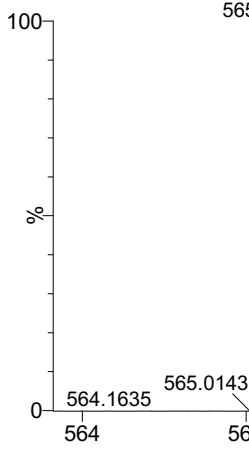

Fig. S21. High Resolution Mass Spectrum of $\mathrm{Bi}(\mathrm{bzq})_{2} \mathrm{Br}(\mathbf{2})$
23-Apr-2019 09:45:10

1: TOF MS ES+

$7.49 \mathrm{e} 12$

Theor $[\mathrm{M}]^{+}: \mathbf{5 6 5 . 1 1 1 8}$

$\operatorname{Exp}[\mathrm{M}]^{+}:+2.5 \mathrm{ppm}(565.1143)$

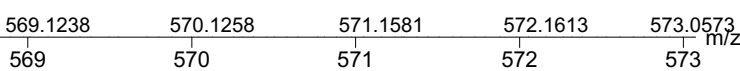




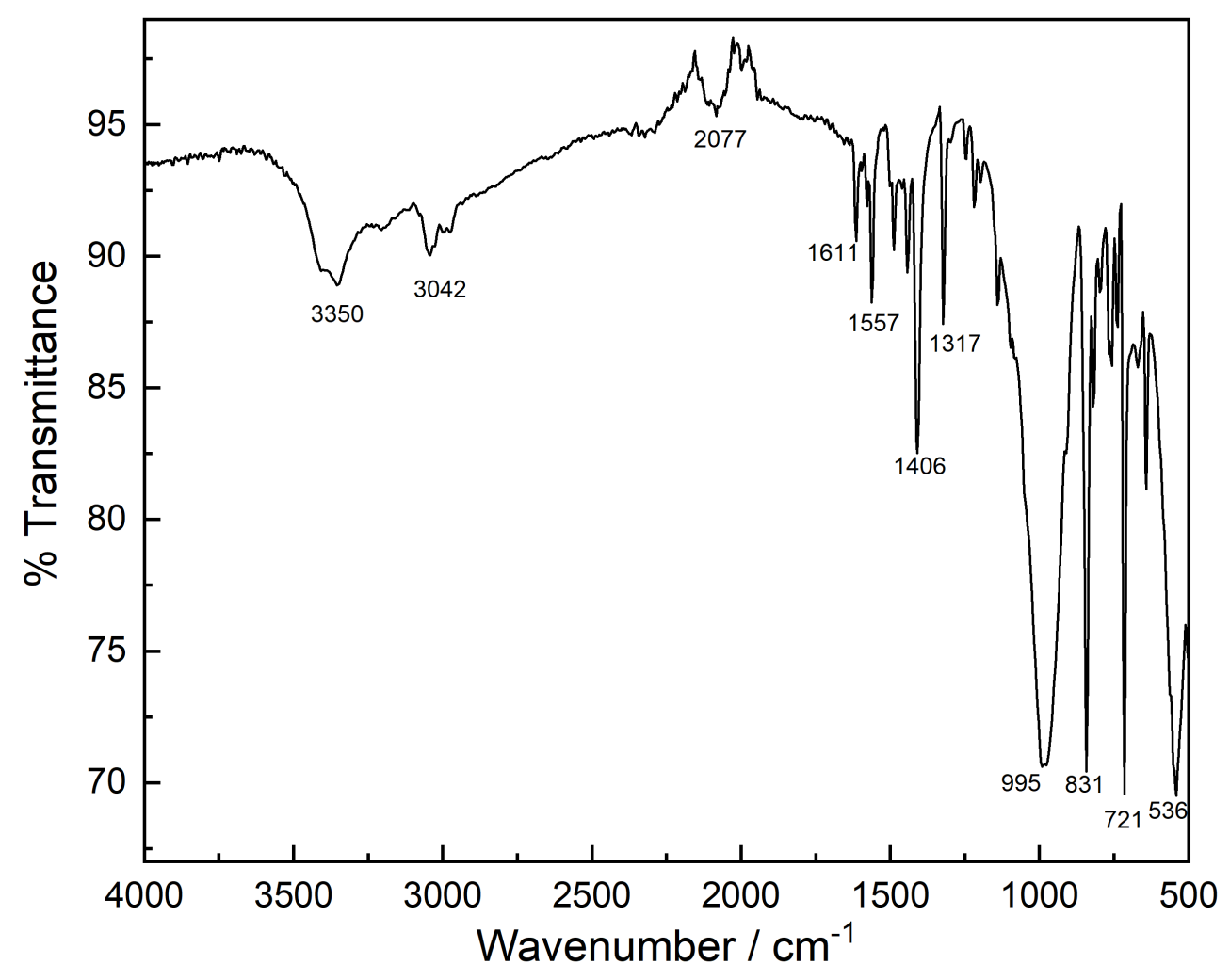

Fig. S22. Infrared Spectrum of $\mathrm{Bi}(\mathrm{bzq})_{2} \mathrm{Br}(2)$ 


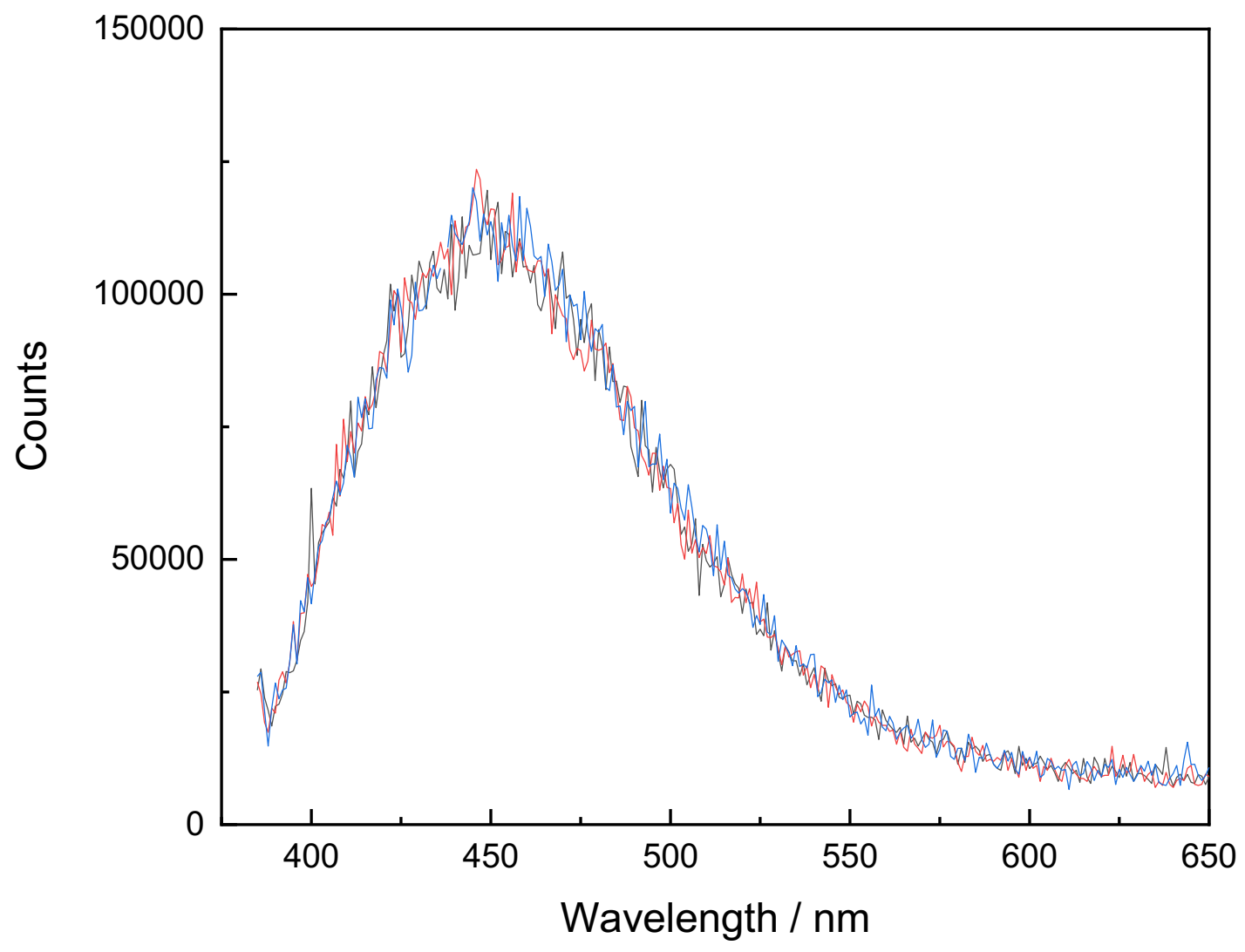

Fig. S23. Steady-State Emission of $\mathrm{Bi}(\mathrm{bzq})_{2}{ }^{ \pm}$

Steady-state emission of $\mathrm{Bi}(\mathrm{bzq})_{2} \mathrm{PF}_{6}$ excited at $375 \mathrm{~nm}$ in $\mathrm{DCM}$. Due to the insolubility of the $\mathrm{Bi}(\mathrm{bzq})_{2} \mathrm{Br}$, the more soluble cation was sought for emission studies. $\mathrm{Bi}(\mathrm{bzq})_{2} \mathrm{Br}$ was partially dissolved in $\mathrm{DCM}$, and a limiting amount of $\mathrm{AgPF}_{6}$ was added to precipitate out a $\mathrm{AgBr}$ salt. Remaining solids were filtered off, and the emission was tested in situ. 


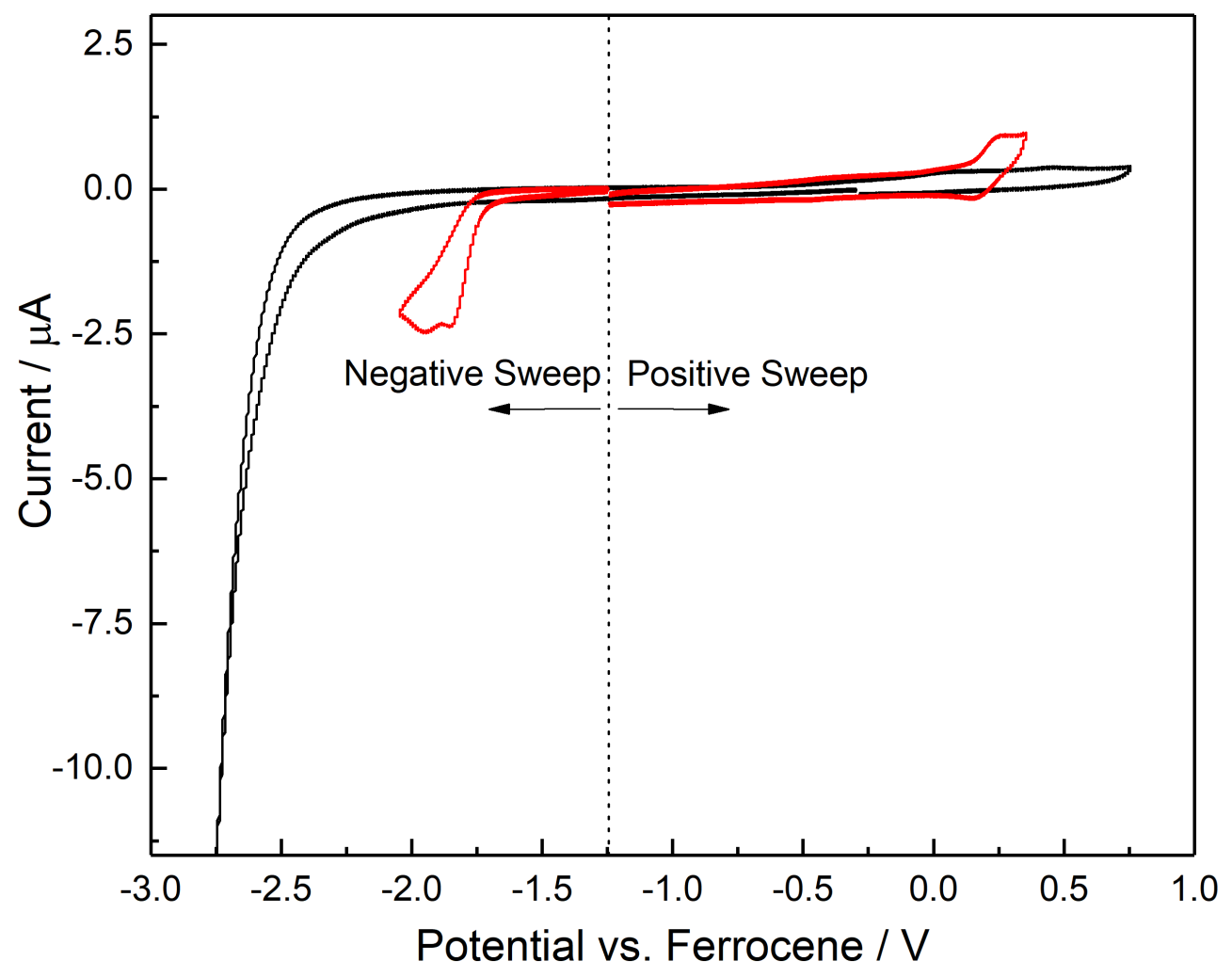

Fig. S24. Cyclic Voltammogram of $\mathrm{Bi}(\mathrm{bzq})_{2} \mathrm{Br}(2)$

Two scans were collected for the DCM solvent background (black) and $\mathrm{Bi}(\mathrm{bzq})_{2} \mathrm{Br}$ (red) scanning positively and negatively from the $-1.25 \mathrm{~V}$ versus ferrocene. The positive and negative sweeps were overlaid to show the oxidation and reduction activity of $\mathrm{Bi}(\mathrm{bzq})_{2} \mathrm{Br}$. 


\section{Benzo[h]quinoline Characterization}

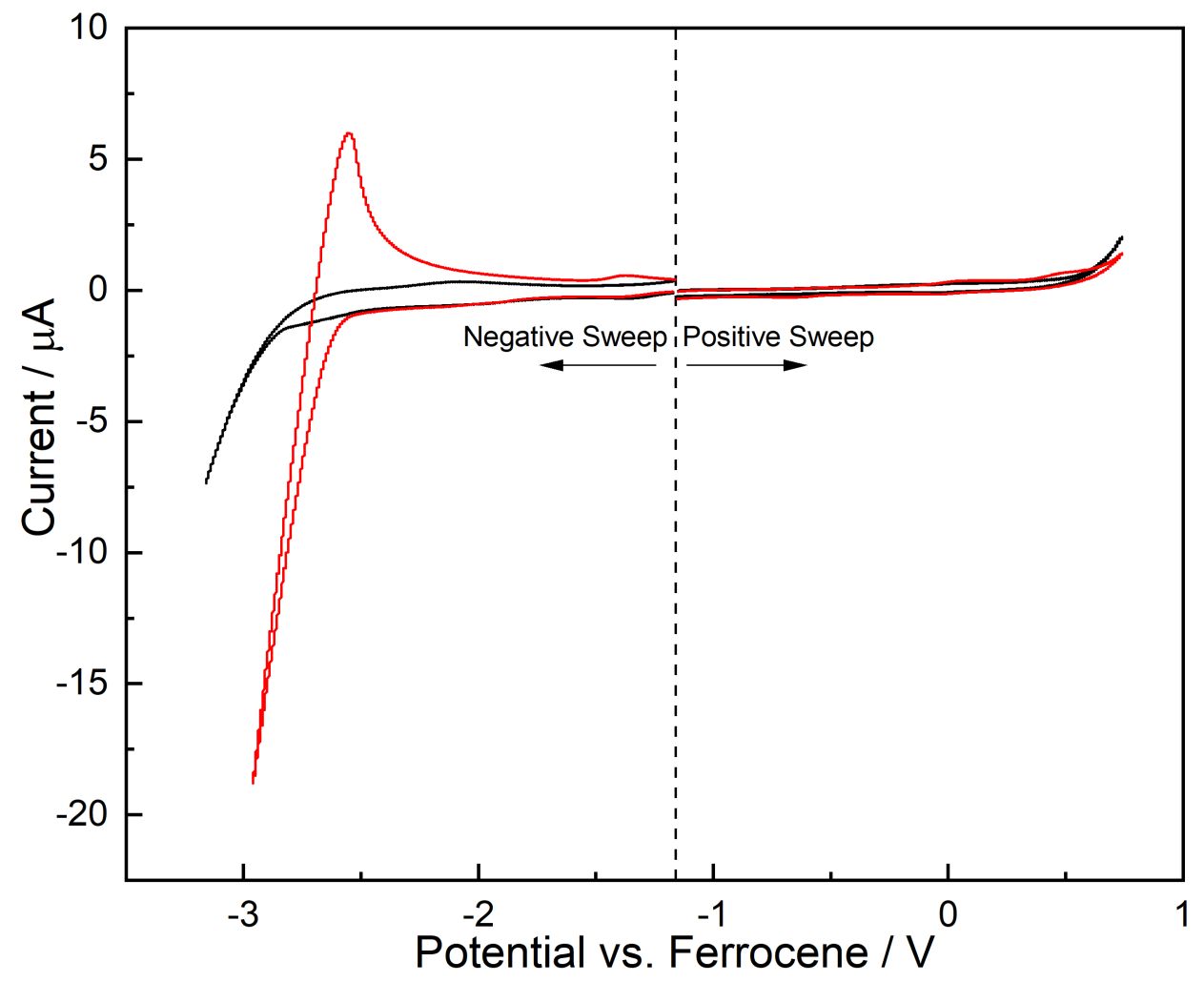

Fig. S25. Cyclic Voltammogram of bzq

Fig. S11. Two scans were collected for the background (black) and bzq-H (red). The positive and negative sweeps were overlaid to show the oxidation and reduction activity of bzq- $\mathrm{H}$. 


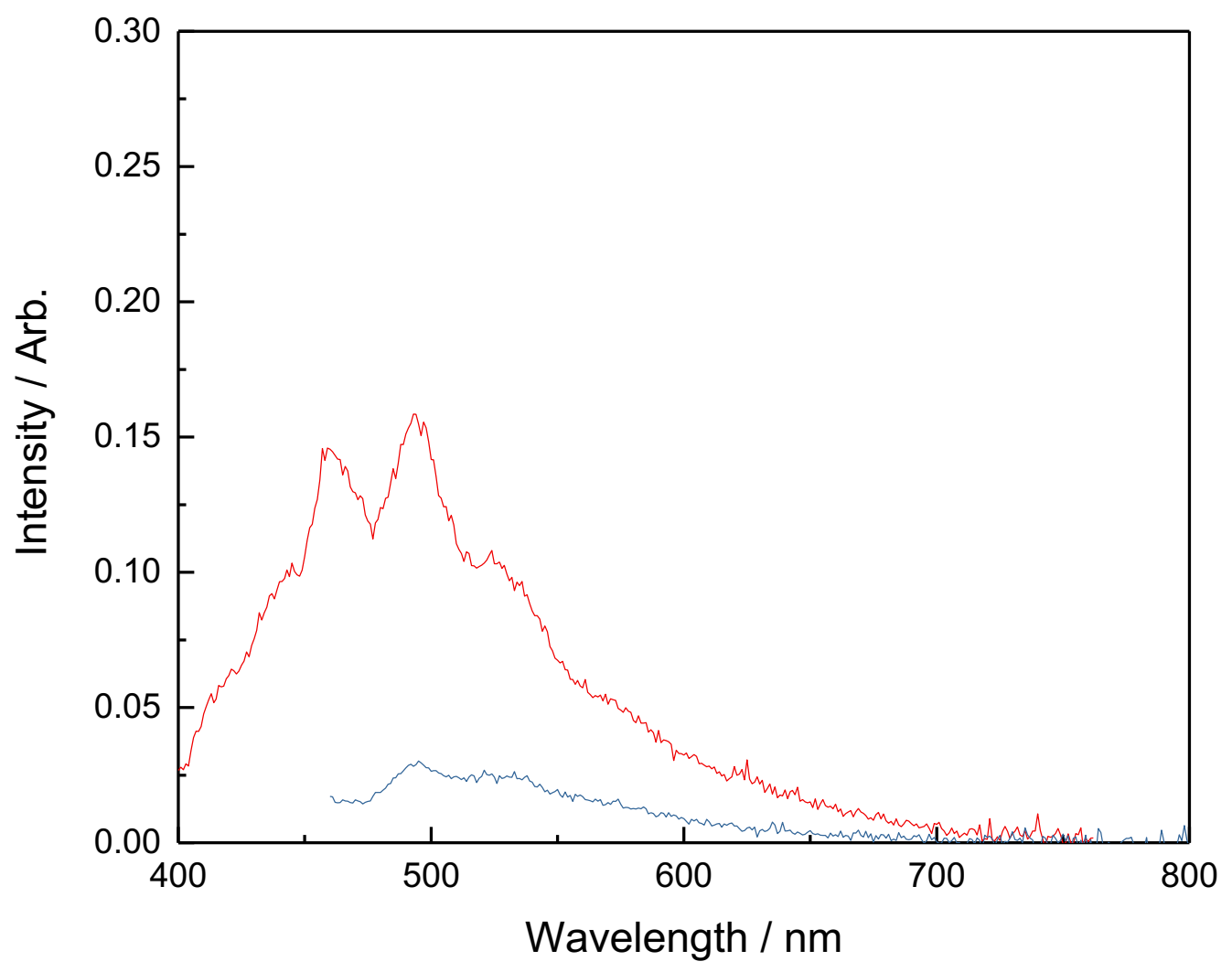

Fig. S26. Emission Spectrum of bza

Emission scans of protonated benzo[h]quinoline excited at $390 \mathrm{~nm}$ (red) and $450 \mathrm{~nm}$ (blue), taken in THF. 


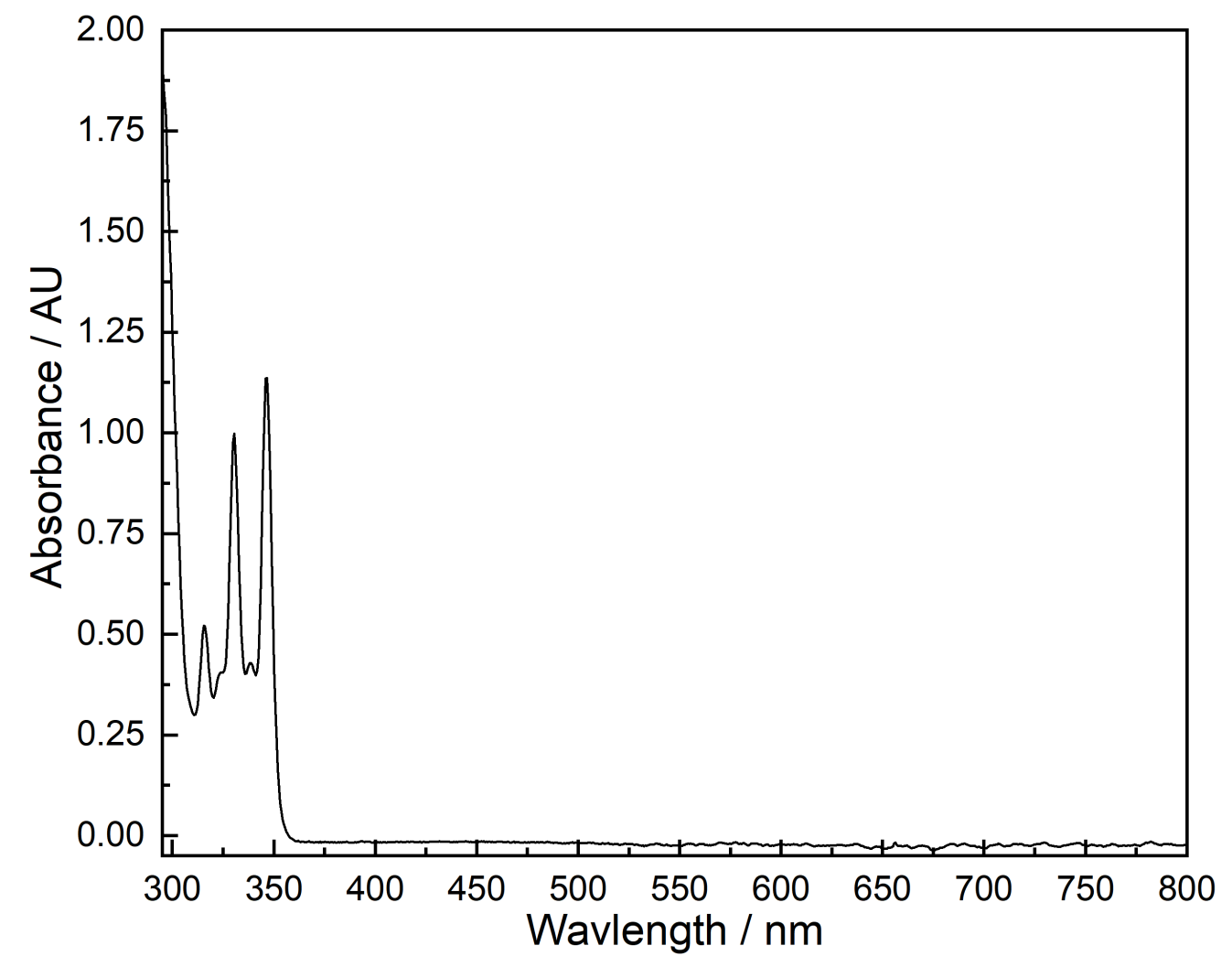

Fig. S27. Absorption Spectrum of bzq

Above is the absorption spectrum for bzq- $\mathrm{H}$, collected in THF. 


\section{Crystallographic Information}

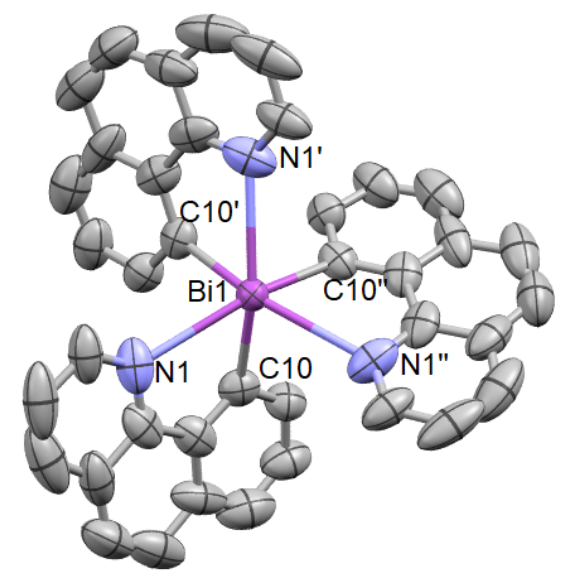

Table S14. Selected bond lengths of compound 1. The tris-ligated complex is formed through growing the crystal structure, so all bond lengths and angles are equivalent on every ligand.

\begin{tabular}{cc}
\hline Bonds & Bond Length $(\mathbf{A})$ \\
\hline $\mathrm{Bi}(1)-\mathrm{C}(10)$ & $2.291(7)$ \\
$\mathrm{Bi}(1)-\mathrm{N}(1)$ & $2.882(6)$
\end{tabular}

Table S15. Below is a table of relevant crystal structure data for compound 1. The tris-ligated complex is formed through growing the crystal structure, so all bond lengths and angles are equivalent on every ligand. Please see picture above table S5 for atom labels.

\begin{tabular}{cc}
\hline Angles & Bond Angles $\left(^{\circ}\right)$ \\
\hline $\mathrm{C}(10)-\mathrm{Bi}(1)-\mathrm{N}(1)$ & $65.7(2)$ \\
$\mathrm{C}(10)-\mathrm{Bi}(1)-\mathrm{C}(10)^{\prime}$ & $93.1(2)$ \\
$\mathrm{C}(10)-\mathrm{Bi}(1)-\mathrm{C}(10)^{\prime}$ & $93.1(2)$ \\
$\mathrm{N}(1)-\mathrm{Bi}(1)-\mathrm{N}(1)^{\prime}$ & $116.68(7)$ \\
\hline
\end{tabular}



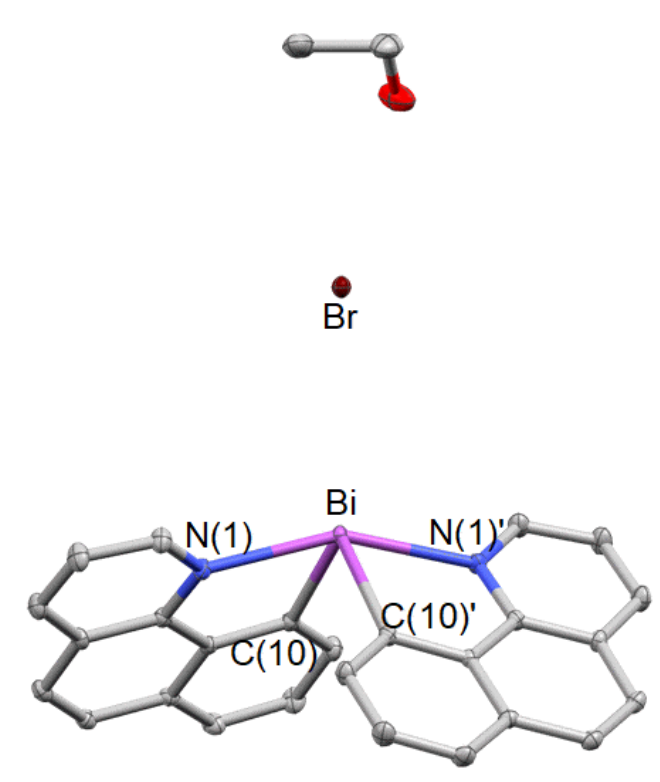

Table S16. Selected bond lengths for compound 2. The bis-ligated complex is formed through growing the crystal structure, so all bond lengths and angles are equivalent on every ligand.

\begin{tabular}{cc}
\hline Bonds & Bond Length $(\AA)$ \\
\hline $\mathrm{Bi}(1)-\mathrm{C}(10)$ & $2.24(1)$ \\
$\mathrm{Bi}(1)-\mathrm{N}(1)$ & $2.43(1)$
\end{tabular}

Table S17. Above is a table of relevant crystal structure data for compound 2 . The tris-ligated complex is formed through growing the crystal structure, so all bond lengths and angles are equivalent on every ligand. Please see picture above table S5 for atom labels.

\begin{tabular}{cc}
\hline Angles & Bond Angles $\left(^{\circ}\right)$ \\
\hline $\mathrm{C}(10)-\mathrm{Bi}(1)-\mathrm{N}(1)$ & $73.6(4)$ \\
$\mathrm{C}(10)-\mathrm{Bi}(1)-\mathrm{C}(10)^{\prime}$ & $93.3(6)$ \\
$\mathrm{N}(1)-\mathrm{Bi}(1)-\mathrm{N}(1)^{\prime}$ & $155.4(5)$ \\
\hline
\end{tabular}


Crystal data for $\mathrm{Bi}(\mathrm{bzq})_{3}$ and $\mathrm{Bi}(\mathrm{bzq})_{2} \mathrm{Br}$

\begin{tabular}{|c|c|c|}
\hline & $\mathrm{Bi}(\mathrm{bzq})_{3}$ & $\mathrm{Bi}(\mathbf{b z q})_{2} \mathrm{Br}$ \\
\hline Formula & $\mathrm{C}_{39} \mathrm{H}_{24} \mathrm{BiN}_{3}$ & $\mathrm{C}_{28} \mathrm{H}_{22} \mathrm{BiBrN}_{2} \mathrm{O}$ \\
\hline fw, g/mol & 743.60 & 691.36 \\
\hline Temperature & $99.99 \mathrm{~K}$ & $107.2 \mathrm{~K}$ \\
\hline cryst. syst. & Cubic & Monoclinic \\
\hline space group & $\mathrm{Pa}-3$ & $\mathrm{C} 2 / \mathrm{c}$ \\
\hline color & Yellow & Yellow \\
\hline $\mathrm{a}(\AA)$ & $18.5262(8)$ & $16.9422(10)$ \\
\hline $\mathrm{b}(\AA)$ & $18.5262(8)$ & $13.2492(10)$ \\
\hline $\mathrm{c}(\AA)$ & $18.5262(8)$ & $12.9069(11)$ \\
\hline$\alpha(\mathrm{deg})$ & 90 & 90 \\
\hline$\beta(\mathrm{deg})$ & 90 & $127.142(4)$ \\
\hline$\gamma(\mathrm{deg})$ & 90 & 90 \\
\hline$V\left(\AA^{3}\right)$ & $6358.6(8)$ & $2309.5(3)$ \\
\hline$Z$ & 8 & 4 \\
\hline no. refl. & 83010 & 82041 \\
\hline unique refl. & 2425 & 6223 \\
\hline $\mathrm{R}_{\text {int }}$ & 0.0680 & 0.0501 \\
\hline $\mathrm{R}^{a}{ }^{a}$ (all data) & 0.0780 & 0.0206 \\
\hline$w \mathrm{R} 2^{b}$ (all data) & 0.0933 & 0.0374 \\
\hline $\mathrm{R} 1[(\mathrm{I}>2 \sigma)]$ & 0.0390 & 0.0162 \\
\hline$w \mathrm{R} 2[\mathrm{I}>2 \sigma)]$ & 0.0933 & 0.0365 \\
\hline $\mathrm{GOF}^{c}$ & 1.044 & 1.056 \\
\hline
\end{tabular}

\section{References:}

(1) Rurack, K.; Spieles, M. Fluorescence Quantum Yields of a Series of Red and Near-Infrared Dyes Emitting at 600-1000 Nm. Anal. Chem. 2011, 83 (4), 1232-1242. https://doi.org/10.1021/ac101329h.

(2) Kira, A.; Thomas, J. K. Equilibriums between Triplet States of Aromatic Hydrocarbons. J. Phys. Chem. 1974, 78 (2), 196-199. https://doi.org/10.1021/j100595a022. 